\title{
Une formule de Plancherel pour l'algèbre de Hecke d'un groupe réductif $p$-adique
}

Volker Heiermann

Résumé. Nous montrons un théorème de Paley-Wiener matriciel pour l'algèbre de Hecke d'un groupe réductif $p$-adique. La preuve est basée sur une analogue de la formule de Plancherel.

Mathematics Subject Classification (2000). Primary 22E35, Secondary 11F70 22 E50.

Mots-clés. Théorème de Paley-Wiener matriciel, formule de Plancherel, algèbre de Hecke, représentations d'un groupe réductif $p$-adique.

Fixons un corps local non archimédien $F$. Soit $G$ l'ensemble des points $F$ rationnels d'un groupe réductif connexe $\underline{G}$ défini sur $F$. Fixons un sous-groupe ouvert compact $K$ maximal spécial de $G$. On munit tout sous-groupe algébrique fermé $H$ de $G$ de la mesure de Haar invariante à gauche pour laquelle mes $(H \cap K)=$ 1. Lorsque $M$ est un sous-groupe de Lévi de $G$ (ou plus précisément l'ensemble des points $F$-rationnels d'un facteur de Lévi d'un sous-groupe parabolique de $\underline{G}$ défini sur $F$ ), notons $\mathfrak{X}^{\text {nr }}(M)$ le groupe des caractères non ramifiés de $M$ (défini en 1.2). C'est une variété algébrique complexe isomorphe à $\left(\mathbb{C}^{\times}\right)^{d}$, où $d$ désigne la dimension du tore déployé maximal dans le centre de $M$. Pour une représentation cuspidale irréductible $(\sigma, E)$ de $M$, on notera $\mathcal{O}_{\sigma}=\left\{\sigma \otimes \chi \mid \chi \in \mathfrak{X}^{\mathrm{nr}}(M)\right\}$ son orbite inertielle. L'application $\mathfrak{X}^{\mathrm{nr}}(M) \rightarrow \mathcal{O}_{\sigma}, \chi \mapsto \sigma \otimes \chi$, définit de façon naturelle une structure de variété algébrique complexe sur $\mathcal{O}_{\sigma}$. Une fonction complexe $\varphi$ sur $\mathcal{O}_{\sigma}$ sera dite polynomiale (resp. rationnelle), si la fonction $\chi \mapsto \varphi(\sigma \otimes \chi)$ est polynomiale (resp. rationnelle) sur $\mathfrak{X}^{\mathrm{nr}}(M)$.

Lorsque $P$ est un sous-groupe parabolique de Lévi $M$, on désigne par $i_{P}^{G}$ le foncteur défini par l'induction parabolique unitaire. Si $M$ et $K$ sont en bonne position relative, on définit l'espace $i_{P \cap K}^{K} E$ des applications $f: K \rightarrow E$ invariantes à droite par un sous-groupe ouvert de $K$ et vérifiant $f(m u k)=\sigma(m) f(k)$ pour tout $m \in M \cap K, u \in U \cap K$ et $k \in K$. La restriction à $K$ définit un isomorphisme de $i_{P}^{G} E$ sur $i_{P \cap K}^{K} E$ et l'espace $i_{P \cap K}^{K} E$ ne change pas si on remplace $\sigma$ par un élément de son orbite inertielle $\mathcal{O}_{\sigma}$. Toutes les représentations $i_{P}^{G} \sigma^{\prime}, \sigma^{\prime} \in \mathcal{O}_{\sigma}$, se réalisent donc dans le même espace $i_{P \cap K}^{K} E$. Ceci permet d'introduire la notion naturelle d'une application polynomiale sur $\mathcal{O}_{\sigma}$ à valeurs dans $\operatorname{Hom}\left(i_{P \cap K}^{K} E, i_{P^{\prime} \cap K}^{K} E\right)$ ou dans $i_{P \cap K}^{K} E \otimes i_{P^{\prime} \cap K}^{K} E^{\vee}$ (cf. [W], IV.1 et VI.1), $P^{\prime}$ désignant un deuxième sous- 
groupe parabolique de Lévi $M$.

L'opérateur d'entrelacement $J_{P^{\prime} \mid P}\left(\sigma^{\prime}\right)$ est défini pour $\sigma^{\prime}$ dans un certain ouvert Zariski dense de $\mathcal{O}_{\sigma}$. C'est une application linéaire de $i_{P \cap K}^{K} E$ dans $i_{P^{\prime} \cap K}^{K} E$ qui vérifie $J_{P^{\prime} \mid P}\left(\sigma^{\prime}\right)\left(i_{P}^{G} \sigma^{\prime}\right)(g)=\left(i_{P^{\prime}}^{G} \sigma^{\prime}\right)(g) J_{P^{\prime} \mid P}\left(\sigma^{\prime}\right)$ pour tout $g \in G, \sigma^{\prime} \in \mathcal{O}_{\sigma}$. On a $\left\langle\left(J_{P^{\prime} \mid P}\left(\sigma^{\prime}\right) v\right)(g), e^{\vee}\right\rangle=\int_{U \cap U^{\prime} \backslash U^{\prime}}\left\langle v\left(u^{\prime} g\right), e^{\vee}\right\rangle d u^{\prime}$ pour $v \in i_{P \cap K}^{K} E$ et $e^{\vee} \in E^{\vee}$, si l'intégrale à droite est convergente. L'application $\mathcal{O}_{\sigma} \rightarrow \operatorname{Hom}\left(i_{P \cap K}^{K} E, i_{P^{\prime} \cap K}^{K} E\right), \sigma^{\prime}$ $\mapsto J_{P^{\prime} \mid P}\left(\sigma^{\prime}\right)$, est rationnelle (i.e. il existe une fonction polynomiale $p$ sur $\mathcal{O}_{\sigma}$ telle que l'application $\sigma^{\prime} \mapsto p\left(\sigma^{\prime}\right) J_{P^{\prime} \mid P}\left(\sigma^{\prime}\right)$ soit polynomiale sur $\left.\mathcal{O}_{\sigma}\right)$. Pour la preuve de ces résultats et d'autres propriétés des opérateurs d'entrelacement, nous renvoyons le lecteur à $[\mathrm{W}]$. Remarquons que la plupart des résultats qui y sont exposés sont dus à Harish-Chandra.

Fixons un tore déployé maximal $A_{0}$ de $G$ par rapport auquel $K$ est en bonne position. (Le groupe $K$ est donc le fixateur d'un point spécial de l'appartement associé à $A_{0}$ dans l'immeuble de $G$.) Notons $W^{G}:=W\left(G, A_{0}\right)$ le groupe de Weyl défini relatif à ce tore. Si $M$ est semi-standard (i.e. $M \supseteq A_{0}$ ) et si $w \in W^{G}$, on dispose d'un isomorphisme $\lambda(w): i_{P}^{G} E \rightarrow i_{w P}^{G} w E, v \mapsto v_{w}, v_{w}(g):=v\left(w^{-1} g\right)$, entre les représentations $i_{P}^{G} \sigma$ et $i_{w P}^{G}(w \sigma)$.

Notons $C_{c}^{\infty}(G)$ l'espace des fonctions complexes lisses à support compact sur $G$. Il est bien connu que l'on peut associer à tout élément $f$ de $C_{c}^{\infty}(G)$ un endomorphisme $\left(i_{P}^{G} \sigma\right)(f)$ de l'espace vectoriel $i_{P}^{G} E$. Nous le noterons $\widehat{f}^{G}(P, \sigma)$, i.e. on pose

$$
\widehat{f}^{G}(P, \sigma):=\int_{G} f(g)\left(i_{P}^{G} \sigma\right)(g) d g .
$$

Notre but est le résultat suivant:

(0.1) Théorème. Étant donné pour chaque (classe d'équivalence d'une) représentation cuspidale irréductible $\left(\sigma, E_{\sigma}\right)$ d'un sous-groupe de Lévi semi-standard $M$ de $G$, et tout sous-groupe parabolique $P$ de Lévi $M$, un endomorphisme $\varphi_{P, \sigma}$ de l'espace vectoriel $i_{P \cap K}^{K} E_{\sigma}$ tels que la famille $\left\{\varphi_{P, \sigma}\right\}_{(P, \sigma)}$ vérifie les propriétés suivantes:

1) Pour tout $(P, \sigma)$, l'application $\varphi_{P, \mathcal{O}}: \mathcal{O} \rightarrow \operatorname{End}\left(i_{P \cap K}^{K} E_{\sigma}\right), \sigma^{\prime} \mapsto \varphi_{P, \sigma^{\prime}}$, est polynomiale sur l'orbite inertielle $\mathcal{O}$ de $\sigma$;

2) Il existe un sous-groupe ouvert compact de $G$ par lequel toute composante $\varphi_{P, \sigma}$ est invariante à gauche et à droite;

3) Pour tout $(P, \sigma)$ et tout $w \in W^{G}$, on a $\lambda(w) \circ \varphi_{P, \sigma}=\varphi_{w P w^{-1}, w \sigma} \circ \lambda(w)$;

4) Pour tout $(P, \sigma)$ et tout $\left(P^{\prime}, \sigma\right)$, on a l'identité d'applications rationnelles $J_{P^{\prime} \mid P}(\sigma) \circ \varphi_{P, \sigma}=\varphi_{P^{\prime}, \sigma} \circ J_{P^{\prime} \mid P}(\sigma)$;

alors il existe une fonction $f$ dans $C_{c}^{\infty}(G)$, telle que $\varphi_{P, \sigma}=\widehat{f}^{G}(P, \sigma)$ pour tout $(P, \sigma)$.

Réciproquement, il est bien connu que, pour $f$ dans $C_{c}^{\infty}(G)$, la famille $\left\{\widehat{f}^{G}(P, \sigma)\right\}_{(P, \sigma)}$ vérifie les propriétés 1 ) - 4) du théorème 0.1. (Pour les propriétés 
2) à 4), c'est une vérification directe et immédiate. La propriété 1) résulte par exemple d'arguments de la théorie des $B$-familles de représentations admissibles utilisés dans la partie B de cet article.)

La propriété 2) équivaut à dire que l'image de $\varphi_{P, \mathcal{O}}$ est contenue dans un sousespace de dimension finie de l'image de l'application canonique $i_{P}^{G} E \otimes i_{P}^{G} E^{\vee} \hookrightarrow$ $\operatorname{End}\left(i_{P}^{G} E\right)$, et qu'il n'existe qu'un nombre fini de $(P, \mathcal{O})$ avec $\varphi_{P, \mathcal{O}} \neq 0$ (cf. [W] théorème VIII.1.2).

Notre démonstration de ce théorème est basée sur une analogue de la formule de Plancherel de Harish-Chandra. Elle a donc l'avantage d'expliciter la fonction $f$ du théorème. La preuve utilise le résultat suivant qui sera prouvé dans la partie $\mathrm{B}$ :

(0.2) Proposition. Soit $\left\{\varphi_{P, \mathcal{O}}\right\}_{(P, \mathcal{O})}$ comme dans le théorème. Pour tout $(P, \mathcal{O})$, $i l$ existe une application polynomiale $\xi_{P, \mathcal{O}}: \mathcal{O} \rightarrow i \frac{K}{P} \cap{ }_{K} E_{\mathcal{O}} \otimes i_{P \cap K}^{K} E_{\mathcal{O}}^{\vee}$ (où $E_{\mathcal{O}}:=E_{\sigma}$ pour un $\sigma \in \mathcal{O}$ et où $\bar{P}$ désigne le sous-groupe parabolique opposé de $P$ ) à image dans un espace de dimension finie, telle que

$$
\varphi_{P, \mathcal{O}}(\sigma)=\sum_{w \in W ; w \mathcal{O}=\mathcal{O}}\left(J_{P \mid \overline{w P}}(\sigma) \circ \lambda(w)\right) \otimes\left(J_{P \mid w P}\left(\sigma^{\vee}\right) \circ \lambda(w)\right) \xi_{P, \mathcal{O}}\left(w^{-1} \sigma\right)
$$

pour tout $\sigma \in \mathcal{O}$.

(Ici on a identifié $\varphi_{P, \mathcal{O}}(\sigma) \in \operatorname{End}\left(i_{P \cap K}^{K} E\right)$ à un élément de $i_{P \cap K}^{K} E \otimes i_{P \cap K}^{K} E^{\vee}$.)

Plus précisément, choisissons $\xi_{P, \mathcal{O}}$ et posons $\zeta_{P, \mathcal{O}}(\sigma)=\left(J_{\bar{P} \mid P}(\sigma)^{-1} \otimes 1\right) \xi_{P, \mathcal{O}}(\sigma)$. Pour $\sigma \in \mathcal{O}$, notons $E_{P, \sigma}^{G}$ l'application linéaire qui associe à un élément $v \otimes v^{\vee}$ de $i_{P \cap K}^{K} E_{\mathcal{O}} \otimes i_{P \cap K}^{K} E_{\mathcal{O}}^{\vee}$ la fonction $g \mapsto\left\langle\left(i_{P}^{G} \sigma\right)(g) v, v^{\vee}\right\rangle, g \in G$. Fixons $\sigma \in \mathcal{O}$. Avec $\varphi=\varphi_{P, \mathcal{O}}$, posons

$$
f_{\varphi}(g)=\int_{\operatorname{Re}(\chi)=\mu \gg_{P} 0} E_{P, \sigma \otimes \chi}^{G}\left(\zeta_{P, \mathcal{O}}(\sigma \otimes \chi)\right)\left(g^{-1}\right) d \operatorname{Im}(\chi),
$$

pour $g \in G$. (La partie réelle d'un caractère non ramifié étant définie dans 1.2, la notation $\mu \gg_{P} 0$ est justifiée par le fait que l'on peut trouver $\mu$ dans la chambre de Weyl de $P$ tel que les pôles $\chi$ de la fonction dans l'intégrale vérifient $\left\langle\alpha^{\vee}, \operatorname{Re}(\chi)\right\rangle<$ $\left\langle\alpha^{\vee}, \mu\right\rangle$ pour toute racine $\alpha$ positive pour $P$. Grâce au théorème des résidus, la valeur de l'intégrale ne dépend alors pas du choix de $\mu$ vérifiant cette condition.)

On montrera (cf. A.3.1-2):

1) La fonction $f_{\varphi_{P, \mathcal{O}}}$ ne dépend que de $\varphi_{P, \mathcal{O}}$;

2) La fonction $f_{\varphi_{P, \mathcal{O}}}$ appartient à $C_{c}^{\infty}(G)$;

Notons $[\mathcal{O}]=\left\{w \sigma \mid \sigma \in \mathcal{O}, w \in W^{G}\right\}$ la classe de $W^{G}$-conjugaison de $\mathcal{O}$ et $M$ le sous-groupe de Lévi semi-standard sous-jacent à $\mathcal{O}$. Posons $f_{\varphi_{[\mathcal{O}]}}=$ $c([\mathcal{O}]) \sum_{\left(P^{\prime}, \mathcal{O}^{\prime}\right)} f_{\varphi_{P^{\prime}, \mathcal{O}^{\prime}}}$, où $c([\mathcal{O}])$ est une constante précisée dans 3.2 , la somme 
portant sur les couples $\left(P^{\prime}, \mathcal{O}^{\prime}\right)$ formés d'une orbite inertielle $\mathcal{O}^{\prime}=w \mathcal{O}, w \in W^{G}$, et d'un sous-groupe parabolique de Lévi $w M w^{-1}$. Alors on a par ailleurs

3) $\widehat{f_{\varphi_{[\mathcal{O}]}}}(P, \sigma)=0$ si $\sigma \notin[\mathcal{O}]$;

4) $\widehat{f_{\varphi_{[\mathcal{O}}}}(P, \sigma)=\varphi_{P, \sigma}$ si $\sigma \in[\mathcal{O}]$;

5) $\int_{G} f_{\varphi_{[\mathcal{O}]}}(g) \overline{f_{\varphi_{\left[\mathcal{O}^{\prime}\right]}}(g)} d g=0$ si $[\mathcal{O}] \neq\left[\mathcal{O}^{\prime}\right]$;

6) La fonction $f_{\varphi}=\sum_{[\mathcal{O}]} f_{\varphi_{[\mathcal{O}]}}$ vérifie $\varphi_{P, \sigma}=\widehat{f}(P, \sigma)$ pour tout $(P, \sigma)$.

Cet article est divisé en deux parties. Dans la partie A, nous prouvons tous les résultats annoncés dans l'introduction à l'exception de la proposition 0.2. Sa preuve est le contenu de la partie B. Les deux parties peuvent être lues indépendamment, seules certaines définitions et notations introduites dans la section A.1 seront utilisées sans rappel dans la partie B.

Remarquons que J.N. Bernstein a annoncé une preuve du théorème 0.1 par une méthode différente de la nôtre.

L'essentiel de ce travail a été réalisé alors que l'auteur séjournait à l'Université Paris 7 au sein de l'équipe "Théorie des Groupes". Ce séjour a été financé par une bourse Feodor Lynen de la fondation Alexander von Humboldt en correspondance avec M.-F. Vignéras. Cette bourse comprenait une participation financière de l'Université Paris 7 venant du réseau "Géométrie arithmétique algébrique" soutenu par le programme "Formation et Mobilité des Chercheurs" de l'Union Européenne. Mon tuteur auprès de la fondation Alexander von Humboldt était E.-W. Zink.

Mes remerciements vont par ailleurs tout particulièrement à J.-L. Waldspurger à qui je dois l'idée pour ce travail et qui m’a bien accompagné durant sa réalisation.

Finalement je remercie le rapporteur qui a examiné mon article pour son travail très soigneux.

\section{A. Une analogue de la formule de Plancherel}

1. On garde les notations et définitions de l'introduction. On notera $q$ le cardinal du corps résiduel de $F, v_{F}$ la valuation discrète de $F$ normalisée telle que $v_{F}\left(F^{\times}\right)=$ $\mathbb{Z}$ et $\|_{F}$ la valeur absolue, donnée par $|x|_{F}=q^{-v_{F}(x)}$ pour $x$ dans $F^{\times}$.

Les définitions et notations qui seront introduites dans la suite pour le groupe réductif $G$ et munies du symbôle $G$ seront ensuite utilisées pour tout groupe réductif $M$, en remplaçant $G$ par $M$, sans que cela soit dit explicitement.

1.1 Notons $A_{G}$ le tore déployé maximal dans le centre de $G$ et $G^{\text {der }}$ le groupe dérivé de $G$. Posons $X^{*}(G)=\operatorname{Hom}_{F}\left(G, \mathbb{G}_{m}\right)$ et $X_{*}(S)=\operatorname{Hom}_{F}\left(\mathbb{G}_{m}, S\right)$ lorsque 
$S$ est un tore.

1.1.1 Posons $a_{0}^{*}=X^{*}\left(A_{0}\right) \otimes_{\mathbb{Z}} \mathbb{R}, a_{G}^{*}=X^{*}\left(A_{G}\right) \otimes_{\mathbb{Z}} \mathbb{R}$ et $a_{0}^{G *}=X^{*}\left(A_{0} \cap G^{\text {der }}\right) \otimes_{\mathbb{Z}} \mathbb{R}$. On a une décomposition $a_{0}^{*}=a_{G}^{*} \oplus a_{0}^{G *}$. Lorsque $S$ est un tore déployé dans $A_{0}$, on notera $\Sigma(S)$ l'ensemble des racines pour l'action adjointe de $S$ dans l'algèbre de Lie de $G$. Soit $(P, M)$ un couple parabolique semi-standard, i.e. $P$ est un sous-groupe parabolique de $G, M$ un facteur de Lévi de $P$, et on a $M \supseteq A_{0}$. L'ensemble $\Sigma\left(A_{M}\right)$ est l'ensemble des projections non nulles dans $a_{M}^{*}$ d'éléments de $\Sigma\left(A_{0}\right)$ suivant la décomposition $a_{0}^{*}=a_{M}^{*} \oplus a_{0}^{M *}$. On notera $\Sigma(P)$ l'ensemble des racines pour l'action adjointe de $A_{M}$ dans l'algèbre de Lie du radical unipotent de $P$.

1.1.2 On fixera pour la suite un couple parabolique semi-standard $\left(P_{0}, M_{0}\right)$ avec $P_{0}$ minimal. On a alors $M_{0}=Z_{G}\left(A_{0}\right)$ et $A_{M_{0}}=A_{0}$. L'ensemble $\Sigma\left(A_{0}\right)$ est un système de racines dans $a_{0}^{G *}$. Remarquons que ce système de racines peut ne pas être réduit. Les éléments de $\Sigma\left(P_{0}\right)$ s'identifient aux racines positives dans $\Sigma\left(A_{0}\right)$ pour un certain ordre sur $a_{0}^{G *}$. La base de $\Sigma\left(A_{0}\right)$ correspondant à cet ordre sera noté $\Delta$. Un couple parabolique $(P, M)$ sera dit standard, s'il est semi-standard et $P \supseteq P_{0}$. On a une bijection $\Omega \mapsto\left(P_{\Omega}, M_{\Omega}\right)$ entre les sous-ensembles de $\Delta$ et les couples paraboliques standard, les racines dans $\Sigma\left(A_{0}\right)$ de restriction triviale à $A_{M_{\Omega}}$ étant les combinaisons linéaires de $\Omega$.

1.1.3 Posons $a_{0}=X_{*}\left(A_{0}\right) \otimes_{\mathbb{Z}} \mathbb{R}, a_{G}=X_{*}\left(A_{G}\right) \otimes_{\mathbb{Z}} \mathbb{R}$ et $a_{0}^{G}=X_{*}\left(A_{0} \cap G^{\text {der }}\right) \otimes_{\mathbb{Z}} \mathbb{R}$. Les espaces $a_{0}$ et $a_{0}^{*}$ sont duaux, l'orthogonal de $a_{G}$ dans $a_{0}^{*}$ est $a_{0}^{G *}$ et celui de $a_{0}^{G}$ est $a_{G}^{*}$.

1.1.4 Si $M$ est un sous-groupe de Lévi semi-standard, il existe une notion de coracine $\alpha^{\vee}$ associée à une racine $\alpha \in \Sigma\left(A_{M}\right)$. C'est un élément de $a_{M}$. On en déduit, pour tout sous-groupe parabolique $P$ de Lévi $M$, une notion de chambre de Weyl dans $a_{M}^{*}$ qui est l'ensemble des éléments positifs pour $P$.

1.1.5 On définit une application $H_{0}: M_{0} \rightarrow \operatorname{Hom}\left(X^{*}\left(M_{0}\right), \mathbb{R}\right) \simeq a_{0} \operatorname{par}\left\langle\chi, H_{0}(m)\right\rangle$ $=v_{F}(\chi(m))$. Soit $(P, M)$ un couple parabolique semi-standard. Un élément $a \in A_{M}$ sera dit positif pour $P$, si $\left\langle\alpha, H_{0}(m)\right\rangle \geq 0$ pour tout $\alpha \in \Sigma(P)$. On dira qu'il est strictement positif, si l'on a l'inégalité stricte pour tout $\alpha \in \Sigma(P)$.

1.2 La restriction $X^{*}(G) \rightarrow X^{*}\left(A_{G}\right)$ induit un isomorphisme $X^{*}(G) \otimes_{\mathbb{Z}} \mathbb{R} \rightarrow a_{G}^{*}$. Le groupe $\mathfrak{X}^{\mathrm{nr}}(G)$ des caractères non ramifiés de $G$ est par définition l'image de l'homomorphisme $a_{G, \mathbb{C}}^{*}=a_{G}^{*} \otimes_{\mathbb{R}} \mathbb{C} \rightarrow \operatorname{Hom}\left(G, \mathbb{C}^{\times}\right)$qui associe à $\lambda=\alpha \otimes s$ le caractère $\chi_{\lambda}$ tel que $\chi_{\lambda}(g)=|\alpha(g)|_{F}^{s}$. Son noyau est de la forme $\frac{2 \pi i}{\log q} R_{G}$, où $R_{G}$ désigne un réseau de rang maximal dans $X^{*}(G) \otimes_{\mathbb{Z}} \mathbb{Q}$. L'homomorphisme munit $\mathfrak{X}^{\mathrm{nr}}(G)$ d'une structure de variété algébrique complexe isomorphe à $\left(\mathbb{C}^{\times}\right)^{d}$ avec $d=$ rang de $A_{G}$. Sa restriction à $a_{G}^{*}$ induit un isomorphisme avec le sous-groupe 
des caractères réels à valeurs $>0$. La partie réelle d'un caractère non ramifié $\chi$, noté $\operatorname{Re}(\chi)$, est l'unique élément $\lambda$ de $a_{G}^{*}$ qui vérifie $\chi_{\lambda}=|\chi|$. On notera $\mathfrak{X}_{\mathrm{im}}^{\mathrm{nr}}(G)$ le sous-groupe de $\mathfrak{X}^{\mathrm{nr}}(G)$ formé des $\chi$ tels que $\operatorname{Re}(\chi)=0$.

1.2.1 On munit $\mathfrak{X}_{\mathrm{im}}^{\mathrm{nr}}\left(A_{G}\right)$ de la mesure de Haar de masse totale 1 , et $\mathfrak{X}_{\mathrm{im}}^{\mathrm{nr}}(G)$ de la mesure de Haar pour laquelle la restriction $\mathfrak{X}_{\mathrm{im}}^{\mathrm{nr}}(G) \rightarrow \mathfrak{X}_{\mathrm{im}}^{\mathrm{nr}}\left(A_{G}\right)$ préserve localement les mesures. Lorsque $\sigma$ est une représentation de $G$, on notera $\operatorname{Stab}_{\mathfrak{X}_{\mathrm{im}}^{\mathrm{nr}}(G)}(\sigma)$ le sous-groupe de $\mathfrak{X}_{\mathrm{im}}^{\mathrm{nr}}(G)$, formé des caractères $\chi$ tels que $\sigma \simeq \sigma \otimes \chi$. Ce sousgroupe ne change pas si on remplace $\sigma$ par un autre élément de son orbite inertielle $\mathcal{O}$, ce qui permettra d'écrire $\operatorname{Stab}_{\mathfrak{X}_{\mathrm{im}}^{\mathrm{nr}}(G)}(\mathcal{O})$.

1.2.2 Soit $(P, M)$ un couple parabolique semi-standard. Soit $r$ une fonction rationnelle sur $\mathfrak{X}^{\text {nr }}(M)$. Supposons qu'il existe un nombre fini d'hyperplans de la forme $\left\langle\lambda, \alpha^{\vee}\right\rangle=c$ dans $a_{M}^{*}, \alpha \in \Sigma(P)$, tels que tout pôle $\chi$ de $r$ soit de la forme $\chi=\chi_{\lambda}$ avec $\lambda$ sur un de ces hyperplans. Il résulte du théorème des résidus que l'intégrale $\int_{\mathfrak{X}_{\mathrm{im}}^{\mathrm{ni}}(M)} r\left(\chi_{0} \chi\right) d \chi$ reste constante, si $\chi_{0}$ varie dans l'ouvert de $\mathfrak{X}^{\mathrm{nr}}(M)$ défini par les inégalités $\left\langle\operatorname{Re}(\chi), \alpha^{\vee}\right\rangle\left\langle\left\langle\operatorname{Re}\left(\chi_{0}\right), \alpha^{\vee}\right\rangle, \chi\right.$ parcourant les pôles de $r$, $\alpha \in \Sigma(P)$.

On écrira plus simplement $\int_{\operatorname{Re}(\chi)=\mu \gg_{P} 0} r(\chi) d(\operatorname{Im}(\chi))$ pour la valeur de cette intégrale.

L'expression $\int_{\operatorname{Re}(\chi)=\mu \ll_{P} 0} r(\chi) d(\operatorname{Im}(\chi))$ aura la signification évidente.

1.2.3 Proposition: Soient $D$ un ouvert de $a_{G}^{*}$ et $\psi$ une fonction holomorphe dans l'ouvert de $\mathfrak{X}^{\mathrm{nr}}(G)$ formé des points $\chi$ avec $\operatorname{Re}(\chi) \in D$. Fixons $\mu \in D$.

Alors, pour tout $\chi_{0} \in \mathfrak{X}^{\text {nr }}(G), \operatorname{Re}\left(\chi_{0}\right)=\mu$, on a

$$
\sum_{a \in A_{G} \cap K \backslash A_{G}} \chi_{0}^{-1}(a) \int_{\operatorname{Re}(\chi)=\mu} \psi(\chi) \chi(a) d \operatorname{Im}(\chi)=\sum_{\chi} \psi\left(\chi \chi_{0}\right),
$$

la somme portant sur les élément de $\mathfrak{X}_{\mathrm{im}}^{\mathrm{nr}}(G)$ de restriction triviale à $A_{G}$.

Preuve: Ceci résulte de la théorie de Fourier sur un tore.

1.3 Fixons un sous-groupe de Lévi semi-standard $M$ de $G$. Notons $\mathcal{P}(M)$ l'ensemble des sous-groupes paraboliques $P$ de $G$ de la forme $P=M U$. Fixons une représentation irréductible cuspidale $(\sigma, E)$ de $M$. Soient $P, P^{\prime} \in \mathcal{P}(M)$. Les points, où l'application rationnelle $\mathfrak{X}^{\mathrm{nr}}(M) \rightarrow \operatorname{Hom}\left(i_{P \cap K}^{K} E, i_{P^{\prime} \cap K}^{K} E\right), \chi \mapsto J_{P^{\prime} \mid P}(\sigma \otimes \chi)$, a un pôle ou bien où $J_{P^{\prime} \mid P}(\sigma \otimes \chi)$ n'est pas inversible, sont de la forme $\chi=\chi_{\lambda}$ avec $\lambda$ sur un nombre fini d'hyperplans de $a_{M}^{*}$ de la forme $\left\langle\alpha^{\vee}, \lambda\right\rangle=c, \alpha \in \Sigma\left(P^{\prime}\right) \cap \Sigma(\bar{P})$. Soit $P^{\prime \prime} \in \mathcal{P}(M)$. Il existe une fonction rationnelle $j_{P\left|P^{\prime}\right| P^{\prime \prime}}$ sur l'orbite inertielle $\mathcal{O}$ de $\sigma$, telle que $J_{P \mid P^{\prime}}(\sigma \otimes \chi) J_{P^{\prime} \mid P^{\prime \prime}}(\sigma \otimes \chi)=j_{P\left|P^{\prime}\right| P^{\prime \prime}}(\sigma \otimes \chi) J_{P \mid P^{\prime \prime}}(\sigma \otimes \chi)$ pour tout $\chi$. Si $P^{\prime \prime}=P$, on écrira plus simplement $j_{P \mid P^{\prime}}=j_{P\left|P^{\prime}\right| P^{\prime \prime}}$. L'égalité $j_{P\left|P^{\prime}\right| P^{\prime \prime}}(\sigma \otimes \chi)=1$ vaut si $d\left(P \mid P^{\prime \prime}\right)=d\left(P \mid P^{\prime}\right)+d\left(P^{\prime} \mid P^{\prime \prime}\right), d\left(P \mid P^{\prime \prime}\right)$ désignant le 
nombre d'hyperplans séparant les chambres de Weyl de $a_{M}^{*}$ correspondant à $P$ et $P^{\prime \prime}$ respectivement (cf. [W], IV.1. (12)).

1.3.1 Le théorème suivant est un résultat clé pour la suite. (A notre connaissance il est paru pour la première fois (en tous cas le cas tempéré) dans les papiers de Casselman et dans ceux de Harish-Chandra, sans que nous nous sentions compétent de l'attribuer à l'un ou l'autre. Il nous a semblé que ce que nous faisons relève davantage de Casselman, Harish-Chandra adoptant un point de vue très analytique.)

Soit $\left(P^{\prime}, M^{\prime}\right)$ un couple parabolique semi-standard. Posons $W\left(M, M^{\prime}\right)=$ $W^{M^{\prime}} \backslash\left\{w \in W^{G} \mid w M w^{-1} \subseteq M^{\prime}\right\}$ et identifions ses éléments à certains éléments de $W^{G}$. Les formules qui suivent seront essentiellement indépendantes du choix d'une telle identification. Pour $w \in W\left(M, M^{\prime}\right)$, définissons $P_{w}^{\prime}, \widetilde{P}_{w}^{\prime} \in \mathcal{P}(M)$ par $P_{w}^{\prime}=\left(w^{-1} M^{\prime} w \cap P\right) w^{-1} U^{\prime} w$ et $\widetilde{P}_{w}^{\prime}=\left(w^{-1} M^{\prime} w \cap P\right) w^{-1} \overline{U^{\prime}} w$.

Désignant par $\delta_{P^{\prime}}$ le module de $P^{\prime}$, on définit une constante

$$
\gamma\left(G / M^{\prime}\right):=\int_{\overline{U^{\prime}}} \delta_{P^{\prime}}\left(m_{P^{\prime}}\left(\overline{u^{\prime}}\right)\right) d \overline{u^{\prime}}
$$

où pour tout élément $g$ de $G$, on a noté $g=u_{P^{\prime}}(g) m_{P^{\prime}}(g) k_{P^{\prime}}(g)$ la décomposition correspondant à la décomposition $G=P^{\prime} K$. (Cette définition de $\gamma\left(G / M^{\prime}\right)$ ne dépend pas du choix de $P^{\prime}$ (cf. [W] p. 5, (3)).)

Pour $v \in i_{P \cap K}^{K} E, v^{\vee} \in i_{P \cap K}^{K} E^{\vee}, \chi \in \mathfrak{X}^{\mathrm{nr}}(M)$ et $a \in A_{M^{\prime}}$, posons $c_{P^{\prime} \mid P}(\sigma \otimes$ $\chi, w)\left(v \otimes v^{\vee}\right)(a)=\left\langle i_{w P \cap M^{\prime}}^{M^{\prime}}(w(\sigma \otimes \chi))(a)\left(\lambda(w) J_{P_{w}^{\prime} \mid P}(\sigma \otimes \chi) v\right)_{\mid M^{\prime}},\left(\lambda(w) J_{\widetilde{P}_{w}^{\prime} \mid P}\left(\sigma^{\vee} \otimes\right.\right.\right.$ $\left.\left.\left.\chi^{-1}\right) v^{\vee}\right)_{\mid M^{\prime}}\right\rangle_{M^{\prime}}$

Théorème. Soient $v \in i_{P \cap K}^{K} E$ et $v^{\vee} \in i_{P \cap K}^{K} E^{\vee}$. Il existe $t>0$, de sorte que pour tout $\chi \in \mathfrak{X}^{\mathrm{nr}}(M)$ et tout $a \in A_{M^{\prime}}$ tel que $\left\langle\alpha, H_{0}(a)\right\rangle>t$ pour tout $\alpha \in \Sigma\left(P^{\prime}\right)$, on ait

$$
\left\langle i_{P}^{G}(\sigma \otimes \chi)(a) v, v^{\vee}\right\rangle=\gamma\left(G \mid M^{\prime}\right)^{-1} \delta_{P^{\prime}}^{1 / 2}(a) \sum_{w \in W\left(M, M^{\prime}\right)} c_{P^{\prime} \mid P}(\sigma \otimes \chi, w)\left(v \otimes v^{\vee}\right)(a) .
$$

Preuve: Notons $\langle,\rangle_{P^{\prime}}$ le produit bilinéaire de Casselman (cf. [Cs] proposition 4.2.3 et théorème 4.2.4). Pour $(\pi, V)$ une représentation lisse de $G$, désignons par $\left(\pi_{P^{\prime}}, V_{P^{\prime}}\right)$ la représentation de $M^{\prime}$ dans le module de Jacquet $V_{P^{\prime}}$ de $V$ et par $j_{P^{\prime}}: V \rightarrow V_{P^{\prime}}$ la projection canonique. Il est prouvé dans [W] au cours de la démonstration du lemme VI.2.1 qu'il existe $t>0$, de sorte que pour tout $\chi \in \mathfrak{X}^{\mathrm{nr}}(M)$ et tout $a \in A_{M^{\prime}}$ tel que $\left\langle\alpha, H_{0}(a)\right\rangle>t$ pour tout $\alpha \in \Sigma\left(P^{\prime}\right)$, on ait

$$
\left\langle i_{P}^{G}(\sigma \otimes \chi)(a) v, v^{\vee}\right\rangle=\left\langle i_{P}^{G}(\sigma \otimes \chi)_{P^{\prime}}(a) j_{P}(v), j_{\bar{P}}\left(v^{\vee}\right)\right\rangle_{P^{\prime}}
$$

Tout se ramène donc au calcul du produit bilinéaire de Casselman. Ce calcul est effectué dans [W] relatif au module de Jacquet faible dans la preuve de la 
proposition V.1.1. Celui relatif au module de Jacquet suit les même lignes: d'une caractérisation des produits bilinéaires $M^{\prime}$-invariants sur $\left(i_{P \cap K}^{K} E\right)_{P^{\prime}} \times\left(i_{P \cap K}^{K} E^{\vee}\right) \overline{P^{\prime}}$ analogue au cas tempéré, on déduit l'existence de constants $\gamma(P, w, \sigma \otimes \chi)$, tels que

$$
\left\langle j_{P^{\prime}}(v), j_{\overline{P^{\prime}}}\left(v^{\vee}\right)\right\rangle_{P^{\prime}}=\sum_{w \in W\left(M . M^{\prime}\right)} \gamma(P, w, \sigma \otimes \chi) c_{P^{\prime} \mid P}(\sigma \otimes \chi, w)\left(v \otimes v^{\vee}\right)(1) .
$$

pour tous $v \in V, v^{\vee} \in V^{\vee}$.

On refait alors les arguments de $[\mathrm{W}]$ dans la preuve de la proposition V.1.1 pour montrer que $\gamma(P, w, \sigma \otimes \chi)=\gamma\left(G / M^{\prime}\right)^{-1}$ pour tout $w \in W\left(M, M^{\prime}\right)$.

1.3.2 Le résultat suivant sera utile lors des applications du théorème 1.3.1.

Lemme. Soit $w \in W\left(M, M^{\prime}\right)$. On a

$$
J_{P_{w}^{\prime} \mid P}\left(\sigma^{\prime}\right) J_{\bar{P} \mid P}\left(\sigma^{\prime}\right)^{-1}=j_{P_{w}^{\prime} \mid \bar{P}}\left(\sigma^{\prime}\right)^{-1} J_{P_{w}^{\prime} \mid \bar{P}}\left(\sigma^{\prime}\right)
$$

en tout point $\sigma^{\prime}$ de l'orbite inertielle de $\sigma$ en lequel ces opérateurs sont définis.

Preuve: Par la formule du produit (cf. [W] p. 55), on a

$$
J_{P_{w}^{\prime} \mid \bar{P}}\left(\sigma^{\prime}\right) J_{\bar{P} \mid P}\left(\sigma^{\prime}\right)=j_{P_{w}^{\prime} \mid \bar{P}}\left(\sigma^{\prime}\right) J_{P_{w}^{\prime} \mid P}\left(\sigma^{\prime}\right)
$$

1.4. Soient $(\pi, V)$ et $\left(\pi^{\prime}, V^{\prime}\right)$ deux représentations irréductibles cuspidales de $G$. Supposons que les restrictions à $A_{G}$ de leurs caractères centraux coïncident. Pour $v \in V, v^{\vee} \in V^{\vee}, v^{\prime} \in V^{\prime}$ et $v^{\prime \vee} \in V^{\prime \vee}$, posons

$$
I\left(v, v^{\vee}, v^{\prime}, v^{\prime \vee}\right)=\int_{A_{G} \backslash G}\left\langle\pi(g) v, v^{\vee}\right\rangle\left\langle v^{\prime}, \pi^{\prime \vee}(g) v^{\prime \vee}\right\rangle d g .
$$

Théorème. (cf. [Ca] Theorem 1.3 et [Cs] proposition 5.2.4)

i) Si $\pi \not \pi^{\prime}$, alors $I\left(v, v^{\vee}, v^{\prime}, v^{\prime \vee}\right)=0$ pour tous $v, v^{\vee}$, $v^{\prime}$ et $v^{\prime \vee}$.

ii) Si $(\pi, V)=\left(\pi^{\prime}, V^{\prime}\right)$, il existe un réel $d(\pi)>0$, appelé le degré formel de $\pi$, tel que $I\left(v, v^{\vee}, v^{\prime}, v^{\prime \vee}\right)=d(\pi)^{-1}\left\langle v, v^{\prime \vee}\right\rangle\left\langle v^{\prime}, v^{\vee}\right\rangle$ pour tous $v, v^{\vee}$, $v^{\prime}$ et $v^{\prime \vee}$.

L'expression $I\left(v, v^{\vee}, v^{\prime}, v^{\vee}\right)$ ne change pas, si on tord $\pi$ et $\pi^{\prime}$ par un même caractère non ramifié. On en déduit que le degré formel de $\pi$ reste invariant si on remplace $\pi$ par un élément de son orbite inertielle $\mathcal{O}$. On peut donc poser $d(\mathcal{O}):=d(\pi)$ 
2. Fixons un couple parabolique standard $(P, M)$ de $G$ et une représentation cuspidale unitaire irréductible $(\sigma, E)$ de $M$. Notons $\mathcal{O}$ l'orbite inertielle de $\sigma$. Soit $\xi: \mathcal{O} \rightarrow i \frac{K}{P} \cap K=i_{P \cap K}^{K} E^{\vee}$ une application polynomiale à image dans un espace de dimension finie. Posons $\zeta\left(\sigma^{\prime}\right)=\left(J_{\bar{P} \mid P}\left(\sigma^{\prime}\right)^{-1} \otimes 1\right) \xi\left(\sigma^{\prime}\right)$ pour tout $\sigma^{\prime} \in \mathcal{O}$ et

$$
f_{\zeta}(g)=\int_{\operatorname{Re}(\chi)=\mu \gg_{P} 0} E_{P, \sigma \otimes \chi}^{G}(\zeta(\sigma \otimes \chi))\left(g^{-1}\right) d(\operatorname{Im}(\chi)) .
$$

2.1 Proposition: La fonction $f_{\zeta}$ appartient à $C_{c}^{\infty}(G)$.

Preuve. D'après 1.2 .2 et 1.3 , la fonction $f_{\zeta}$ est bien définie. Il est clair qu'elle est lisse. Il reste donc à montrer que son support est compact. Comme $\xi$ est une somme finie de fonctions $\mathfrak{X}^{\mathrm{nr}}(M) \rightarrow \mathbb{C}, \chi \mapsto p(\chi) v \otimes v^{\vee}$ avec $p$ fonction polynomiale sur $\mathfrak{X}^{\mathrm{nr}}(M)$ et $v \otimes v^{\vee} \in i_{P \cap K}^{K} E \otimes i_{P \cap K}^{K} E^{\vee}$, il suffit de considérer le cas où $\xi$ est une telle fonction.

Par la décomposition de Cartan, on a

$$
G=K M_{0}^{+} K \text { avec } M_{0}^{+}=\left\{m \in M_{0} \mid \forall \alpha \in \Delta\left\langle\alpha, H_{0}(m)\right\rangle \geq 0\right\} \text {. }
$$

Par ailleurs, $K m K=K m^{\prime} K$ si et seulement si $H_{0}(m)=H_{0}\left(m^{\prime}\right)$. Posons $A_{0}^{+}=$ $A_{0} \cap M_{0}^{+}$. On observe que $M_{0}$ est l'ensemble des points $F$-rationnels d'un groupe réductif (défini sur $F$ ) qui est le produit presque direct du tore déployé maximal dans son centre et d'un groupe anisotrope. L'ensemble des points $F$-rationnels de ce tore étant $A_{0}$, il existe un compact $C$ de $A_{0}^{+}$tel que $M_{0}^{+}=C A_{0}^{+}$. Par un argument de $K-C$-finitude - l'ensemble des transformés d'un élément de $V$ par un compact de $G$ engendre un sous-espace de dimension finie de $V$-, il suffit alors de montrer que, pour tout $v \in i_{\bar{P} \cap K}^{K} E$, tout $v^{\vee} \in i_{P \cap K}^{K} E^{\vee}$ et toute fonction polynomiale $p$ sur $\mathfrak{X}^{\mathrm{nr}}(M)$, la fonction sur $A_{0}^{+}$définie par

$$
a \mapsto \int_{\operatorname{Re}(\chi)=\mu \gg_{P} 0} p(\chi)\left\langle i_{P}^{G}(\sigma \otimes \chi)(a) J_{\bar{P} \mid P}(\sigma \otimes \chi)^{-1} v, v^{\vee}\right\rangle d(\operatorname{Im}(\chi))
$$

est à support compact.

Supposons d'abord $G$ semi-simple. Alors $\Delta$ est une base de $a_{0}^{*}$. Pour $\Theta \subseteq \Delta$ et $t^{\prime}, t \geq 0$, posons $A_{0}^{+}\left(\Theta, t, t^{\prime}\right)=\left\{a \in A_{0}^{+} \mid\left\langle\alpha, H_{0}(a)\right\rangle \leq t \forall \alpha \in \Theta\right.$ et $\left\langle\alpha, H_{0}(a)\right\rangle>$ $\left.t^{\prime} \forall \alpha \in \Delta-\Theta\right\}$, et $A_{0}^{+}(\Theta, t):=A_{0}^{+}(\Theta, t, t)$.

On va montrer l'existence d'une fonction $(\Theta, t) \mapsto f(\Theta, t), \Theta \subset \Delta, t \geq 0$, telle que la fonction $\left(^{*}\right)$ soit nulle en tout $a \in A_{0}^{+}(\Theta, t, f(\Theta, t))$. Ceci implique la proposition dans le cas semi-simple: Comme $A_{0}^{+}=\bigcup_{\Theta \subset \Delta} A_{0}^{+}(\Theta, t)$ pour tout $t \geq 0$, il suffit d'en déduire que, pour tout $\Theta \subseteq \Delta$ et tout $\bar{t} \geq 0$, la restriction de $(*)$ à $A_{0}^{+}(\Theta, t)$ est à support compact. 
Effectuons une récurrence décroissante sur $\Theta \subseteq \Delta$. Comme $\Delta$ est une base de $a_{0}^{*}, A_{0}^{+}(\Delta, t)$ est compact pour tout $t \geq 0$. Soit $\Theta \subset \Delta$. Si $\left(^{*}\right)$ est non nulle en $a \in A_{0}^{+}(\Theta, t)$, alors $\Theta^{\prime}=\left\{\alpha \in \Delta \mid\left\langle\alpha, H_{0}(a)\right\rangle \leq f(\Theta, t)\right\}$ contient strictement $\Theta$. Par suite, $a \in \bigcup_{\Theta^{\prime} \supset \Theta} A_{0}^{+}\left(\Theta^{\prime}, f(\Theta, t)\right)$. Or, par hypothèse de récurrence, la restriction de l'application $(*)$ à cette réunion est à support compact.

Fixons $\Theta \subset \Delta, t \geq 0$ et montrons l'existence de $f(\Theta, t)$. Posons $\left(P^{\prime}, M^{\prime}\right)=$ $\left(P_{\Theta}, M_{\Theta}\right)$. Il existe un compact $C_{\Theta} \subset A_{0}, C_{\Theta}^{-1} \subset A_{0}^{+}$, tel que tout élément de $A_{0}^{+}$ puisse s'écrire sous la forme $a=a_{\Theta} a^{\prime} c_{a}$ avec $a_{\Theta} \in A_{M^{\prime}}, a^{\prime} \in A_{0} \cap M^{\prime \text { der }}$ et $c_{a} \in C_{\Theta}$. On a $\left\langle\alpha, H_{0}\left(a^{\prime}\right)\right\rangle \leq 0$ pour tout $\alpha \in \Delta-\Theta$, et l'ensemble des $H_{0}\left(a^{\prime}\right)$ avec $a \in$ $A_{0}^{+}(\Theta, t)$ est fini. En particulier, $a \in A_{0}^{+}\left(\Theta, t, t^{\prime}\right)$ implique $a_{\Theta} \in A_{\Theta} \cap A_{0}^{+}\left(\Theta, t, t^{\prime}\right)$.

Par ailleurs, comme $\alpha(a)=\alpha\left(a a_{\Theta}^{-1}\right)$ pour tout $\alpha \in \Theta$, on en déduit l'existence d'un compact $C_{\Theta}^{\prime}$ de $A_{0}$, tel que $a a_{\Theta}^{-1} \in C_{\Theta}^{\prime}$ pour tout $a \in A_{0}^{+}(\Theta, t)$.

Lorsque $a$ et $\sigma \otimes \chi$ parcourent respectivement $A_{0}^{+}(\Theta, t)$ et l'ensemble des points $J_{\bar{P} \mid P}^{-1}$-réguliers de $\mathcal{O}$, l'ensemble des $i_{P}^{G}(\sigma \otimes \chi)\left(a a_{\Theta}^{-1}\right) J_{\bar{P} \mid P}(\sigma \otimes \chi)^{-1} v$ reste donc dans un espace de dimension finie. Il résulte donc de la formule de Casselman (1.3.1), qu'il existe $t_{0} \geq t$, tel que, pour tout $a \in A_{0}^{+}\left(\Theta, t, t_{0}\right)$, on ait

$$
\begin{aligned}
& \left\langle i_{P}^{G}(\sigma \otimes \chi)(a) J_{\bar{P} \mid P}(\sigma \otimes \chi)^{-1} v, v^{\vee}\right\rangle \\
= & \gamma\left(G \mid M^{\prime}\right)^{-1} \delta_{P^{\prime}}^{1 / 2}(a) \sum_{w \in W\left(M, M^{\prime}\right)} c_{P^{\prime} \mid P}(\sigma \otimes \chi, w)\left(J_{\bar{P} \mid P}(\sigma \otimes \chi)^{-1} v \otimes v^{\vee}\right)(a) .
\end{aligned}
$$

En particulier, le coefficient matriciel est nul, si $W\left(M, M^{\prime}\right)=\emptyset$.

L'étude de $(*)$ se ramène donc à celle de

$$
a \mapsto \int_{\operatorname{Re}(\chi)=\mu \gg_{P} 0} p(\chi) c_{P^{\prime} \mid P}(\sigma \otimes \chi, w)\left(J_{\bar{P} \mid P}(\sigma \otimes \chi)^{-1} v \otimes v^{\vee}\right)(a) d(\operatorname{Im}(\chi))
$$

pour tout $w \in W\left(M, M^{\prime}\right)$. Fixons $w \in W\left(M, M^{\prime}\right)$.

Pour $a \in A_{0}^{+}$, notons $r_{a}$ la fonction rationnelle $\chi \mapsto p(\chi) c_{P^{\prime} \mid P}(\sigma \otimes \chi, w)\left(J_{\bar{P} \mid P}(\sigma \otimes\right.$ $\left.\chi)^{-1} v \otimes v^{\vee}\right)\left(a^{\prime} c_{a}\right)$ définie sur $\mathfrak{X}^{\mathrm{nr}}(M)$. Comme l'ensemble formé des $a a_{\Theta}^{-1}=a^{\prime} c_{a}^{\prime}$, $a \in A_{0}^{+}(\Theta, t)$, est contenu dans le compact $C_{\Theta}^{\prime}$, il résulte d'un argument de $C_{\Theta^{-}}^{\prime}$ finitude que les fonctions $r_{a}, a \in A_{0}^{+}(\Theta, t)$, sont en nombre fini.

Par ce qui précède et après avoir effectué le changement de base $\chi \mapsto w^{-1} \chi$, l'étude de $(* *)$ se ramène à celle de

$$
a \mapsto \int_{\operatorname{Re}(\chi)=w \mu} \chi\left(a_{\Theta}\right) r_{a}\left(w^{-1} \chi\right) d(\operatorname{Im}(\chi)),
$$

où $\mu$ a été choisi suffisamment positif dans la chambre de Weyl de $P$ dans $a_{M}^{*}$.

On déduit de 1.3 et de 1.3.2 que les pôles des fonctions rationnelles $\chi \mapsto$ $r_{a}\left(w^{-1} \chi\right), a \in A_{0}^{+}(\Theta, t)$, (qui sont en nombre fini) sont de la forme $\chi_{\lambda}$ avec 
$\lambda$ sur un nombre fini d'hyperplans de $a_{M}^{*}$ de la forme $\left\langle\alpha^{\vee}, \lambda\right\rangle=c$ avec $\alpha \in$ $\Sigma\left(w P w^{-1}\right) \cap \Sigma\left(w P_{w}^{\prime} w^{-1}\right)$.

A l'aide de la décomposition $a_{w M w^{-1}}^{*}=a_{M^{\prime}}^{*} \oplus a_{w M w^{-1}}^{M^{\prime}{ }^{*}}$, on définit $\chi_{\mu^{\prime}} \in$ $\mathfrak{X}^{\mathrm{nr}}\left(w M w^{-1}\right)$ pour $\mu^{\prime} \in a_{M^{\prime}}^{*}$. Supposons $\mu^{\prime}$ dans la chambre de Weyl positive de $P^{\prime}$ dans $a_{M^{\prime}}^{*}$. Soit $\alpha \in \Sigma\left(w P w^{-1}\right) \cap \Sigma\left(w P_{w}^{\prime} w^{-1}\right)$. Alors, ou bien $\alpha \in \Sigma\left(w P w^{-1} \cap M^{\prime}\right)$ ou bien $\alpha_{\mid A_{M^{\prime}}} \in \Sigma\left(P^{\prime}\right)$. Dans le premier cas, $\left\langle\mu^{\prime}, \alpha^{\vee}\right\rangle=0$, alors que $\left\langle\mu^{\prime}, \alpha^{\vee}\right\rangle>0$ dans le deuxième. D'autre part, $\left\langle w \mu, \alpha^{\vee}\right\rangle \gg 0$, puisque $\alpha \in \Sigma\left(w P w^{-1}\right)$ et $\mu \gg 0$. L'intégrale dans (***) ne change donc pas de valeur pour $a \in A_{0}^{+}(\Theta, t)$, si on remplace $w \mu$ par $w \mu+\mu^{\prime}$ avec $\mu^{\prime}$ dans la chambre de Weyl positive de $P^{\prime}$ dans $a_{M^{\prime}}^{*}$.

L'ensemble des fonctions rationnelles $\chi \mapsto r_{a}\left(w^{-1} \chi\right), a \in A_{0}^{+}(\Theta, t)$, étant fini, on peut choisir $t_{w} \geq t_{0}$ tel que, pour tout $a \in A_{0}^{+}\left(\Theta, t, t_{w}\right), \chi \mapsto \chi\left(a_{\Theta}\right)$ soit à décroissance rapide par rapport à $\chi \mapsto r_{a}\left(w^{-1} \chi\right)$, lorsque $\operatorname{Re}\left(\chi_{\mid A_{M^{\prime}}}\right)$ devient très positif dans la chambre de Weyl de $P^{\prime}$ dans $a_{M^{\prime}}^{*}$.

On a donc vu que, pour $a \in A_{0}^{+}\left(\Theta, t, t_{w}\right)$, l'intégrale dans (***) reste invariante si on remplace $w \mu$ par $w \mu+\mu^{\prime}, \mu^{\prime} \in a_{M^{\prime}}^{*}$ et $\mu^{\prime}>_{P^{\prime}} 0$, alors que la fonction à l'intérieur de l'intégrale converge vers 0 si $\mu^{\prime}$ devient très positif dans la chambre de Weyl de $P^{\prime}$. Ceci prouve que l'expression $(* * *)$ est nulle en tout $a \in A_{0}^{+}\left(\Theta, t, t_{w}\right)$.

On pourra alors prendre pour $f(\Theta, t)$ le plus grand des $t_{w}$.

Considérons maintenant le cas d'un groupe réductif qui n'est pas semi-simple. On a $A_{0}=A_{G}\left(A_{0} \cap G^{\text {der }}\right) C^{\prime}$ avec $C^{\prime}$ compact. Les morphismes de restriction donnent lieu à une suite exacte $0 \rightarrow \mathfrak{X}^{\mathrm{nr}}(G) \times X \rightarrow \mathfrak{X}^{\mathrm{nr}}(M) \rightarrow \mathfrak{X}^{\mathrm{nr}}\left(M \cap G^{\text {der }}\right) \rightarrow 0$, où $X$ désigne un sous-ensemble fini de $\mathfrak{X}_{\mathrm{im}}^{\text {nr }}(M)$ formé de caractères de restriction triviale à $A_{G}\left(M \cap G^{\text {der }}\right)$. On identifie $\mathfrak{X}_{\mathrm{im}}^{\text {nr }}(G)$ à un sous-groupe de $\mathfrak{X}_{\mathrm{im}}^{\mathrm{nr}}(M)$ au moyen de ce morphisme. On a donc un isomorphisme $\mathfrak{X}_{\mathrm{im}}^{\mathrm{nr}}(M) /\left(\mathfrak{X}_{\mathrm{im}}^{\mathrm{nr}}(G) \times X\right) \rightarrow$ $\mathfrak{X}_{\mathrm{im}}^{\mathrm{nr}}\left(M \cap G^{\text {der }}\right)$.

Choisissons $\mu$ suffisamment positif dans la chambre de Weyl de $P$ dans $a_{M}^{*}$ et tel que $\langle\mu, H\rangle=0$ pour $H \in a_{G}$. Par ce qui précède et un argument de $C^{\prime}$-finitude, l'étude de $\left(^{*}\right)$ se ramène à celle de

$$
\begin{aligned}
\int_{\chi_{\mu} \mathfrak{X}_{\mathrm{im}}^{\mathrm{nr}}\left(M \cap G^{\mathrm{der}}\right)} & \int_{\mathfrak{X}_{\mathrm{im}}^{\mathrm{nr}}(G) \times X} p\left(\chi^{\prime} \chi_{G}\right) \chi_{G}\left(a_{G}\right) \\
& \left\langle i_{P}^{G}\left(\sigma \otimes \chi^{\prime} \chi_{G}\right)\left(a^{\prime}\right) J_{\bar{P} \mid P}\left(\sigma \otimes \chi^{\prime} \chi_{G}\right)^{-1} v, v^{\vee}\right\rangle d\left(\operatorname{Im}\left(\chi_{G}\right)\right) d\left(\operatorname{Im}\left(\chi^{\prime}\right)\right)
\end{aligned}
$$

pour $\left(a_{G}, a^{\prime}\right)$ dans $A_{G} \times\left(A_{0}^{+} \cap G^{\text {der }}\right)$. Il reste donc à montrer l'existence de compacts $C_{G}$ et $C_{0}$ de $A_{G}$ et $A_{0}^{+} \cap G^{\text {der }}$ respectivement, tels que (\#) soit nul si $\left(a_{G}, a^{\prime}\right) \notin$ $C_{G} \times C_{0}$.

Remarquons d'abord que, si $a^{\prime} \in A_{0}^{+} \cap G^{\text {der }}$,

$$
\begin{aligned}
& \left\langle i_{P}^{G}\left(\sigma \otimes \chi^{\prime} \chi_{G}\right)\left(a^{\prime}\right) J_{\bar{P} \mid P}\left(\sigma \otimes \chi^{\prime} \chi_{G}\right)^{-1} v, v^{\vee}\right\rangle \\
= & \left\langle i_{P \cap G^{\text {der }}}^{\text {der }}\left(\sigma \otimes \chi^{\prime}\right)\left(a^{\prime}\right) J_{\bar{P} \cap G^{\text {der }} \mid P \cap G^{\text {der }}}\left(\sigma \otimes \chi^{\prime}\right)^{-1} v_{\mid G^{\text {der }}}, v_{\mid G^{\text {der }}}^{\vee}\right\rangle,
\end{aligned}
$$


et que l'on peut remplacer ci-dessus $\int_{\mathfrak{X}_{\mathrm{im}}^{\mathrm{nr}}(G) \times X}$ par $|X| \int_{\mathfrak{X}_{\mathrm{im}}^{\mathrm{nr}}(G)}$. Comme $\chi^{\prime} \mapsto$ $\int_{\mathfrak{X}_{\mathrm{im}}^{\mathrm{nr}}(G)} p\left(\chi^{\prime} \chi_{G}\right) \chi_{G}\left(a_{G}\right) d\left(\operatorname{Im}\left(\chi_{G}\right)\right)$ est une application polynomiale sur $\mathfrak{X}^{\mathrm{nr}}(M \cap$ $\left.G^{\text {der }}\right)$ et que $M \cap G^{\text {der }}$ est un sous-groupe de Lévi semi-standard du groupe semisimple $G^{\text {der }}$, l'existence de $C_{0}$ résulte du cas semi-simple considéré précédemment. L'intégrale sur $\mathfrak{X}_{\mathrm{im}}^{\mathrm{nr}}(G)$ portant sur une fonction polynomiale en $\chi_{G}$, l'existence de $C_{G}$ est immédiate.

2.2 Lorsque $f$ est un élément de $C_{c}^{\infty}(G)$ et que $P^{\prime}=M^{\prime} U^{\prime}$ est un sous-groupe parabolique de $G$, posons

$$
f_{P^{\prime}}\left(m^{\prime}\right)=\delta_{P^{\prime}}\left(m^{\prime}\right)^{1 / 2} \int_{U^{\prime}} f\left(m^{\prime} u^{\prime}\right) d u^{\prime} \quad \text { pour } m^{\prime} \in M^{\prime} .
$$

2.2.1 Lemme. (cf. [S] p.109) On a $f_{P^{\prime}} \in C_{c}^{\infty}\left(M^{\prime}\right)$.

2.2.2 Lemme. Soit $(\pi, V)$ une représentation lisse de $G$ et $v \in V$. Si $P^{\prime}=M^{\prime} U^{\prime}$ est un sous-groupe parabolique et $H$ un sous-groupe ouvert compact de $G$ qui laisse $v$ invariant et qui admet une décomposition d'Iwahori $H=\left(U^{\prime} \cap H\right)\left(M^{\prime} \cap H\right)\left(\overline{U^{\prime}} \cap\right.$ $H)$ par rapport au couple parabolique $\left(P^{\prime}, M^{\prime}\right)$, alors on a

$$
\int_{U^{\prime} \cap H} \pi\left(u^{\prime} a\right) v d u^{\prime}=\frac{m e s\left(U^{\prime} \cap H\right)}{m e s(H)} \int_{H} \pi(h a) v d h .
$$

pour tout $a \in A_{M^{\prime}}$ strictement positif pour $P^{\prime}$. En particulier, l'élément de $V$ égal à cette intégrale est invariant par $H$.

Preuve. Notons $u_{P^{\prime}}(h) m_{P^{\prime}}(h) u_{\overline{P^{\prime}}}(h)$ la décomposition d'un élément $h \in H$ selon la décomposition $H=\left(H \cap U^{\prime}\right)\left(H \cap M^{\prime}\right)\left(H \cap \overline{U^{\prime}}\right)$. Comme, par choix de $a \in A_{M^{\prime}}$, $a^{-1}\left(H \cap \overline{U^{\prime}}\right) a \subseteq H \cap \overline{U^{\prime}}$, on trouve grâce à l'invariance de $v$ par $H$ que $\pi(h a) v=$ $\pi\left(u_{P^{\prime}}(h) a\right) v$. L'égalité du lemme en suit par intégration partielle.

2.2.3 Proposition. Soit $\left(P^{\prime}, M^{\prime}\right)$ un couple parabolique semi-standard, et supposons

$\operatorname{dim} M^{\prime} \leq \operatorname{dim} M$. Alors on a

(i) $\left(\bar{f}_{\zeta}\right)_{P^{\prime}}=0$ si $M^{\prime}$ et $M$ ne sont pas conjugués;

(ii)

$$
\gamma(G \mid M)\left(f_{\zeta}\right)_{P^{\prime}}\left(m^{\prime}\right)=\sum_{w \in W(M, M)} \int_{\operatorname{Re}(w \chi)=\mu_{w} \gg_{P^{\prime}} 0}
$$

$E_{M, w(\sigma \otimes \chi)}^{M}\left(\left(\lambda(w) J_{P_{w}^{\prime} \mid \bar{P}}(\sigma \otimes \chi) \otimes \lambda(w) J_{P_{w}^{\prime} \mid P}\left(\sigma^{\vee} \otimes \chi^{-1}\right)\right) \xi(\sigma \otimes \chi)\right)\left(m^{\prime-1}\right) d I m(\chi)$

si $M^{\prime}=M$. 
Preuve. Comme $\xi$ est une combinaison linéaire d'applications de la forme $\chi \mapsto$ $p(\chi) v \otimes v^{\vee}$, il suffit de montrer la proposition dans le cas où $\xi$ est une telle fonction.

Soit $H$ un sous-groupe ouvert compact de $K$, admettant une décomposition d'Iwahori relative à $\left(P^{\prime}, M^{\prime}\right)$ et laissant $v$ et $v^{\vee}$ invariant. Soit $a \in A_{M^{\prime}}$ strictement positif pour $P^{\prime}$. Posons $U_{0}^{\prime}=H \cap U^{\prime}$. On a $U^{\prime}=\bigcup_{l=0}^{\infty} a^{-l} U_{0}^{\prime} a^{l}$. Par suite,

$$
\begin{aligned}
& \delta_{P^{\prime}}\left(m^{\prime}\right)^{-1 / 2}\left(f_{\zeta}\right)_{P^{\prime}}\left(m^{\prime}\right) \\
&= \int_{U^{\prime}} f_{\zeta}\left(m^{\prime} u^{\prime}\right) d u^{\prime} \\
&= \lim _{l \rightarrow \infty} \int_{a^{-l} U_{0}^{\prime} a^{l}} \int_{\operatorname{Re}(\chi)=\mu \gg_{P} 0} E_{P, \sigma \otimes \chi}^{G}(\zeta(\sigma \otimes \chi))\left(u^{\prime-1} m^{\prime-1}\right) d I m(\chi) d u^{\prime} \\
&= \lim _{l \rightarrow \infty} \delta_{P^{\prime}}\left(a^{l}\right)^{-1} \int_{U_{0}^{\prime}} \int_{\operatorname{Re}(\chi)=\mu \gg_{P} 0} p(\chi) \\
&\left\langle i_{P}^{G}(\sigma \otimes \chi)\left(a^{l}\right) J_{\bar{P} \mid P}(\sigma \otimes \chi)^{-1} i \frac{G}{P}(\sigma \otimes \chi)\left(m^{\prime-1}\right) v, i_{P}^{G}\left(\sigma^{\vee} \otimes \chi^{-1}\right)\left(u^{\prime} a^{l}\right) v^{\vee}\right\rangle d \operatorname{Im}(\chi) d u^{\prime} .
\end{aligned}
$$

En posant $v_{m^{\prime}}=i \frac{G}{P}(\sigma \otimes \chi)\left(m^{\prime-1}\right) v$ et $v_{l}^{\vee}=\int_{U_{0}^{\prime}} i_{P}^{G}\left(\sigma^{\vee} \otimes \chi^{-1}\right)\left(u^{\prime} a^{l}\right) v^{\vee} d u^{\prime}$, ceci devient à l'aide du théorème de Fubini égal à

$$
\lim _{l \rightarrow \infty} \delta_{P^{\prime}}(a)^{-l} \int_{\operatorname{Re}(\chi)=\mu \gg_{P} 0} p(\chi)\left\langle i_{P}^{G}(\sigma \otimes \chi)\left(a^{l}\right) J_{\bar{P} \mid P}(\sigma \otimes \chi)^{-1} v_{m^{\prime}}, v_{l}^{\vee}\right\rangle d \operatorname{Im}(\chi) .
$$

Il résulte du lemme 2.2.2 que $v_{l}^{\vee}$ reste dans un espace de dimension finie pour $l \gg 0$. On peut donc appliquer le théorème 1.3.1, et on trouve

$$
\begin{aligned}
& \left\langle i_{P}^{G}(\sigma \otimes \chi)\left(a^{l}\right) J_{\bar{P} \mid P}(\sigma \otimes \chi)^{-1} v_{m^{\prime}}, v_{l}^{\vee}\right\rangle \\
= & \gamma\left(G \mid M^{\prime}\right)^{-1} \delta_{P^{\prime}}\left(a^{l}\right)^{1 / 2} \sum_{w \in W\left(M, M^{\prime}\right)} c_{P^{\prime} \mid P}(\sigma \otimes \chi, w)\left(J_{\bar{P} \mid P}(\sigma \otimes \chi)^{-1} v_{m^{\prime}} \otimes v_{l}^{\vee}\right)\left(a^{l}\right)
\end{aligned}
$$

pour $l$ assez grand.

Le (i) de la proposition en résulte aussitôt. Supposons dans la suite $M^{\prime}=M$.

Observons que

$$
\begin{aligned}
& \left(\lambda(w) J_{\widetilde{P}_{w}^{\prime} \mid P}\left(\sigma^{\vee} \otimes \chi^{-1}\right) v_{l}^{\vee}\right)(1) \\
= & \int_{U_{0}^{\prime}}\left(J_{\widetilde{P}_{w}^{\prime} \mid P}\left(\sigma^{\vee} \otimes \chi^{-1}\right) i_{P}^{G}\left(\sigma^{\vee} \otimes \chi^{-1}\right)\left(u^{\prime} a^{l}\right) v^{\vee}\right)\left(w^{-1}\right) d u^{\prime} \\
= & \int_{U_{0}^{\prime}}\left(J_{\widetilde{P}_{w}^{\prime} \mid P}\left(\sigma^{\vee} \otimes \chi^{-1}\right) v^{\vee}\right)\left(w^{-1} u^{\prime} a^{l}\right) d u^{\prime} \\
= & \delta_{P^{\prime}}\left(a^{l}\right)^{1 / 2} w\left(\sigma^{\vee} \otimes \chi^{-1}\right)\left(a^{l}\right) \int_{a^{-l} U_{0}^{\prime} a^{l}}\left(J_{\widetilde{P}_{w}^{\prime} \mid P}\left(\sigma^{\vee} \otimes \chi^{-1}\right) v^{\vee}\right)\left(w^{-1} u^{\prime}\right) d u^{\prime} .
\end{aligned}
$$


Par suite,

$$
\begin{aligned}
& \delta_{P^{\prime}}\left(m^{\prime}\right)^{-1 / 2}\left(f_{\zeta}\right)_{P^{\prime}}\left(m^{\prime}\right) \\
= & \gamma\left(G \mid M^{\prime}\right)^{-1} \lim _{l \rightarrow \infty} \sum_{w \in W(M, M)} \int_{\operatorname{Re}(\chi)=\mu \gg_{P} 0} p(\chi) \\
& \left\langle\left(\lambda(w) J_{P_{w}^{\prime} \mid P}(\sigma \otimes \chi) J_{\bar{P} \mid P}(\sigma \otimes \chi)^{-1} v_{m^{\prime}}\right)(1),\right. \\
& \left.\int_{a^{-l} U_{0}^{\prime} a^{l}}\left(J_{\widetilde{P}_{w}^{\prime} \mid P}\left(\sigma^{\vee} \otimes \chi^{-1}\right) v^{\vee}\right)\left(w^{-1} u^{\prime}\right) d u^{\prime}\right\rangle d \operatorname{Im}(\chi) .
\end{aligned}
$$

Fixons $w \in W(M, M)$ et calculons la limite correspondante. Il résulte de 1.3 et de 1.3.2 qu'il existe un nombre fini d'hyperplans de la forme $\left\langle\lambda, \alpha^{\vee}\right\rangle=c$, $\alpha \in \Sigma(P) \cap \Sigma\left(P_{w}^{\prime}\right)$, tels que tout pôle de la fonction dans l'intégrale soit de la forme $\chi_{\lambda}$ avec $\lambda$ sur un de ces hyperplans. On peut donc remplacer ci-dessus $\mu \gg_{P} 0$ par $\mu_{w} \gg_{P_{w}^{\prime}}$ 0. Or, alors $\lim _{l \rightarrow \infty} \int_{a^{-l} U_{0}^{\prime} a^{l}}\left(J_{\widetilde{P}_{w}^{\prime} \mid P}\left(\sigma^{\vee} \otimes \chi^{-1}\right) v^{\vee}\right)\left(w^{-1} u^{\prime}\right) d u^{\prime}=$ $\left(\lambda(w) J_{P_{w}^{\prime} \mid \widetilde{P}_{w}^{\prime}}\left(\sigma^{\vee} \otimes \chi^{-1}\right) J_{\widetilde{P}_{w}^{\prime} \mid P}\left(\sigma^{\vee} \otimes \chi^{-1}\right) v^{\vee}\right)(1)$. En appliquant 1.3.2 et la formule de produit pour les opérateurs d'entrelacement, on en déduit que le terme correspondant à $w$ dans la somme ci-dessus est égal à

$\int_{\operatorname{Re}(\chi)=\mu_{w} \gg P_{P_{w}^{\prime}} 0} p(\chi)\left\langle\left(\lambda(w) J_{P_{w}^{\prime} \mid \bar{P}}(\sigma \otimes \chi) v_{m^{\prime}}\right)(1),\left(\lambda(w) J_{P_{w}^{\prime} \mid P}\left(\sigma^{\vee} \otimes \chi^{-1}\right) v^{\vee}\right)(1)\right\rangle d \operatorname{Im}(\chi)$,

d'où la formule (ii).

2.3 Proposition. Soit $\left(\sigma^{\prime}, E^{\prime}\right)$ une représentation irréductible cuspidale d'un sous-groupe de Lévi semi-standard $M^{\prime}$ de dimension inférieure ou égale à celle de $M$. Soit $P^{\prime} \in \mathcal{P}\left(M^{\prime}\right)$. Alors on a

(i) $\widehat{f}_{\zeta}\left(\sigma^{\prime}, P^{\prime}\right)=0$ si $M^{\prime}$ et $M$ ne sont pas conjugués;

(ii)

$$
\begin{aligned}
& \gamma(G \mid M)\left|\operatorname{Stab}_{\mathfrak{X}_{\mathrm{im}}^{\mathrm{nr}}(M)}\left(\sigma^{\prime}\right)\right|^{-1} \widehat{f}_{\zeta}\left(\sigma^{\prime}, P^{\prime}\right) \\
= & d\left(\sigma^{\prime}\right)^{-1} \sum_{w \in W(M, M), \sigma^{\prime} \in w \mathcal{O}}\left(J_{P^{\prime} \mid \overline{w P}}\left(\sigma^{\prime}\right) \lambda(w) \otimes J_{P^{\prime} \mid w P}\left(\sigma^{\prime \vee}\right) \lambda(w)\right) \xi\left(w^{-1} \sigma^{\prime}\right)
\end{aligned}
$$

si $M^{\prime}=M$.

Pour la preuve de cette proposition, on utilisera les deux lemmes suivants, où $\lambda$ et $\rho$ désignent respectivement l'action par translations à gauche et à droite de $G$ sur $C_{c}^{\infty}(G)$. 
2.3.1 Lemme. Soit $\left(\sigma^{\prime}, E^{\prime}\right)$ une représentation cuspidale de $M^{\prime}, P^{\prime} \in \mathcal{P}\left(M^{\prime}\right)$. Alors on a $\widehat{f}_{\zeta}^{G}\left(\sigma^{\prime}, P^{\prime}\right)(g, h)=\left(\lambda(g) \rho(h) f_{\zeta}\right) \widehat{P}^{\prime} M^{\prime}\left(\sigma^{\prime}, M^{\prime}\right)$.

Preuve. Il suffit de combiner les lemmes VII.1.2 et VII.1.1 (i) de [W] qui y sont prouvés par des arguments qui se généralisent des représentations de carré intégrable aux représentations cuspidales (voir également la remarque au sujet de la preuve de la proposition 2.4), puisque $f_{\zeta}$ est à support compact.

2.3.2 Lemme. On a $\left(\lambda(g) \rho(h) f_{\zeta}\right)=f_{\zeta^{\prime}}$, où $\zeta^{\prime}(\sigma \otimes \chi)=\left(i_{P}^{G}(\sigma \otimes \chi) \otimes i_{P}^{G}\left(\sigma^{\vee} \otimes\right.\right.$ $\left.\left.\chi^{-1}\right)\right)(g, h) \zeta(\sigma \otimes \chi)$.

Preuve. (de la proposition)

La partie (i) de la proposition est une conséquence immédiate des lemmes 2.2.3 (i) et 2.3.1.

Pour prouver la partie (ii), écrivons $\xi(\sigma \otimes \chi)=\sum_{i \in I} p_{i}(\sigma \otimes \chi) v_{i} \otimes v_{i}^{\vee}$ avec $I$ un ensemble fini, $v_{i} \otimes v_{i}^{\vee} \in i_{P \cap K}^{K} E \otimes i_{P \cap K}^{K} E^{\vee}$, et $p_{i}$ une fonction polynomiale en $\chi \in \mathfrak{X}^{\mathrm{nr}}(M)$.

Supposons $M^{\prime}=M$. A l'aide de 2.2.3 (ii), on trouve avec $e^{\prime} \in E^{\prime}$ et $e^{\prime \vee} \in E^{\prime \vee}$,

$$
\begin{aligned}
& \gamma(G \mid M)\left\langle\left(f_{\zeta}\right){\widehat{P^{\prime}}}^{M}\left(\sigma^{\prime}, M\right) e^{\prime}, e^{\prime \vee}\right\rangle \\
= & \sum_{i \in I} \sum_{w \in W(M, M)} \int_{M} \int_{\operatorname{Re}(w \chi)=\mu_{w} \gg P^{\prime} 0} p_{i}(\sigma \otimes \chi) \\
& \left\langle\left(\lambda(w) J_{P_{w}^{\prime} \mid \bar{P}}(\sigma \otimes \chi) v_{i}\right)(1), w(\sigma \otimes \chi)^{\vee}(m)\left(\lambda(w) J_{P_{w}^{\prime} \mid P}\left(\sigma^{\vee} \otimes \chi^{-1}\right) v_{i}^{\vee}\right)(1)\right\rangle d \operatorname{Im}(\chi) \\
& \left\langle\sigma^{\prime}(m) e^{\prime}, e^{\prime \vee}\right\rangle d m \\
= & \sum_{i \in I} \sum_{w \in W(M, M)} \int_{A_{M} \backslash M}\left\langle\sigma^{\prime}(m) e^{\prime}, e^{\prime \vee}\right\rangle \int_{A_{M} \cap K \backslash A_{M}} \int_{\operatorname{Re}(w \chi)=-\mu_{w} \ll P^{\prime} 0} p_{i}\left(\sigma \otimes \chi^{-1}\right) \\
& \left\langle\left(\lambda(w) J_{P_{w}^{\prime} \mid \bar{P}^{\prime}}\left(\sigma \otimes \chi^{-1}\right) v_{i}\right)(1), w\left(\sigma \otimes \chi^{-1}\right)^{\vee}(m)\left(\lambda(w) J_{P_{w}^{\prime} \mid P}\left(\sigma^{\vee} \otimes \chi\right) v_{i}^{\vee}\right)(1)\right\rangle \\
& \chi_{\sigma^{\prime}}(a) w\left(\chi \chi_{\sigma}^{-1}\right)(a)\left(\int_{A_{M} \cap K}\left(\chi_{\sigma^{\prime}}\left(w \chi_{\sigma}\right)^{-1}\right)\left(\epsilon_{a}\right) d \epsilon_{a}\right) d \operatorname{Im}(\chi) d a d m .
\end{aligned}
$$

Comme $A_{M} \cap K$ est compact de mesure 1, l'intégrale sur $A_{M} \cap K$ n'est non nulle que si $\chi_{\sigma^{\prime} \mid A_{M} \cap K}=w \chi_{\sigma \mid A_{M} \cap K}$, et sa valeur est alors 1 . Dans ce cas, $\chi_{\sigma^{\prime}}\left(w \chi_{\sigma}\right)^{-1}$ est la restriction à $A_{M}$ d'un certain élément $w \chi_{w}$ de $\mathfrak{X}^{\mathrm{nr}}(M)$.

A l'aide de la théorie de Fourier sur un tore (cf. 1.2.3), on trouve alors 


$$
\begin{gathered}
=\sum_{i \in I} \sum_{w \in W(M, M)} \sum_{\chi \in \operatorname{ker}\left(\mathfrak{X}_{\mathrm{im}}^{\mathrm{nr}}(M) \rightarrow \mathfrak{X}_{\mathrm{im}}^{\mathrm{nr}}\left(A_{M}\right)\right)} p_{i}\left(\sigma \otimes \chi_{w} \chi\right) \int_{A_{M} \backslash M}\left\langle\sigma^{\prime}\left(m^{\prime}\right) e^{\prime}, e^{\vee \vee}\right\rangle \\
\left\langle\left(\lambda(w) J_{P_{w}^{\prime} \mid \bar{P}}\left(\sigma \otimes \chi_{w} \chi\right) v_{i}\right)(1),\right. \\
\left.w\left(\sigma \otimes \chi_{w} \chi\right)^{\vee}(m)\left(\lambda(w) J_{P_{w}^{\prime} \mid P}\left(\sigma^{\vee} \otimes \chi_{w}^{-1} \chi^{-1}\right) v_{i}^{\vee}\right)(1)\right\rangle d m \\
=d\left(\sigma^{\prime}\right)^{-1}\left|\operatorname{Stab}_{\mathcal{X}_{\mathrm{im}}^{\mathrm{nn}}(G)}\left(\sigma^{\prime}\right)\right| \\
\sum_{i \in I} \sum_{w \in W(M, M), w^{-1} \sigma^{\prime} \in \mathcal{O}} p_{i}\left(w^{-1} \sigma^{\prime}\right)\left\langle\left(\lambda(w) J_{P_{w}^{\prime}} \mid P^{\prime}\left(w^{-1} \sigma^{\prime}\right) v_{i}\right)(1), e^{\prime \vee}\right\rangle \\
\left\langle e^{\prime},\left(\lambda(w) J_{P_{w}^{\prime} \mid P}\left(w^{-1} \sigma^{\prime \vee}\right) v_{i}^{\vee}\right)(1)\right\rangle
\end{gathered}
$$

par 1.4, si $\operatorname{Re}\left(w \chi_{w}\right)<_{P^{\prime}}-\mu_{w}$ pour tout $w$. Comme les deux applications sont rationnelles sur l'orbite inertielle de $\sigma^{\prime}$, on a l'égalité partout.

A l'aide des lemmes 2.2.1, 2.2.3, 2.3.1, et 2.3.2, on en déduit le résultat énoncé.

2.4 Proposition. Soit $\mathcal{O}^{\prime}$ une orbite inertielle définie relative à un sous-groupe de Lévi semi-standard de $G$. Supposons que $\mathcal{O}^{\prime}$ et $\mathcal{O}$ ne soient pas conjuguées. Soit $\zeta^{\prime}$ une application rationnelle sur $\mathcal{O}^{\prime}$ vérifiant les propriétés analogues à celles de $\zeta$ relatives à $\mathcal{O}^{\prime}$.

Alors on a

$$
\int_{G} f_{\zeta}(g) \overline{f_{\zeta^{\prime}}(g)} d g=0
$$

Preuve. Suite à la proposition 2.3 , les arguments dans la démonstration de la proposition VII.2.2 dans [W] se généralisent sans problème, après avoir remarqué que, si $\sigma$ est une représentation unitaire, alors, pour tout $v \in i_{P \cap K}^{K} E$ et tout $v^{\vee} \in$ $i_{P \cap K}^{K} E^{\vee}$, il existe $v_{1} \in i_{P \cap K}^{K} E$ et $v_{1}^{\vee} \in i_{P \cap K}^{K} E^{\vee}$, tels que $\overline{\left\langle i_{P}^{G}(\sigma \otimes \chi)(g) v, v^{\vee}\right\rangle}=$ $\left\langle i_{P}^{G}\left(\sigma \otimes \bar{\chi}^{-1}\right)\left(g^{-1}\right) v_{1}, v_{1}^{\vee}\right\rangle$ pour tout $\chi \in \mathfrak{X}^{\mathrm{nr}}(M)$ et tout $g \in G$.

2.4.1 Corollaire. Soit $\left(\sigma^{\prime}, E^{\prime}\right)$ une représentation irréductible cuspidale d'un sous-groupe de Lévi $M^{\prime}$ de G. Supposons que $\sigma^{\prime}$ ne soit conjuguée à aucun élément de $\mathcal{O}$.

$$
\text { Alors on a } \widehat{f}_{\zeta}\left(\sigma^{\prime}, P^{\prime}\right)=0 \text {. }
$$

Preuve. Notons $\mathcal{O}^{\prime}$ l'orbite inertielle de $\sigma^{\prime}$. Comme $\widehat{f}_{\zeta}\left(., P^{\prime}\right)$ est polynomiale sur $\mathcal{O}^{\prime}$, il suffit de prouver le corollaire pour $\sigma^{\prime}$ unitaire. Choisissons une fonction polynomiale non identiquement nulle $j_{1}$ sur $\mathcal{O}^{\prime}$, telle que l'opérateur rationnel $j_{1} J_{P \mid \bar{P}}$ soit régulier. On va montrer que $\left\langle\widehat{f}_{\zeta}\left(\sigma^{\prime}, P^{\prime}\right) v^{\prime}, v^{\prime \vee}\right\rangle=0$ pour tout $v^{\prime} \otimes v^{\prime \vee} \in$ $i_{P^{\prime} \cap K}^{K} E^{\prime} \otimes i_{P^{\prime} \cap K}^{K} E^{\prime \vee}$. 
Par la remarque dans la preuve de la proposition 2.4, on peut choisir $v_{1}^{\prime} \otimes v_{1}^{\prime \vee} \in$ $i_{P^{\prime} \cap K}^{K} E^{\prime} \otimes i_{P^{\prime} \cap K}^{K} E^{\prime \vee}$, tel que $\overline{\left\langle i_{P}^{G}\left(\sigma^{\prime} \otimes{\overline{\chi^{\prime}}}^{-1}\right)(g) v^{\prime}, v^{\prime \vee}\right\rangle}=\left\langle i_{P}^{G}\left(\sigma^{\prime} \otimes \chi^{\prime}\right)\left(g^{-1}\right) v_{1}^{\prime}, v_{1}^{\prime \vee}\right\rangle$. Soit $p$ une fonction polynomiale sur $\mathcal{O}^{\prime}$. Posons $\xi^{\prime}\left(\sigma^{\prime} \otimes \chi^{\prime}\right)=p\left(\sigma^{\prime} \otimes \chi^{\prime}\right) j_{1}\left(\sigma^{\prime} \otimes\right.$ $\left.\chi^{\prime}\right)\left(J_{P \mid \bar{P}}\left(\sigma^{\prime} \otimes \chi^{\prime}\right) v_{1}^{\prime} \otimes v_{1}^{\prime \vee}\right)$ pour $\chi^{\prime} \in \mathfrak{X}^{\mathrm{nr}}\left(M^{\prime}\right)$. Par choix de $j_{1}$, cette application est polynomiale en $\chi^{\prime}$.

Posons $\zeta^{\prime}\left(\sigma^{\prime} \otimes \chi^{\prime}\right):=\left(J_{\bar{P} \mid P}^{-1}\left(\sigma^{\prime} \otimes \chi^{\prime}\right) \otimes 1\right) \xi^{\prime}\left(\sigma^{\prime} \otimes \chi^{\prime}\right)=p\left(\sigma^{\prime} \otimes \chi^{\prime}\right) j_{1}\left(\sigma^{\prime} \otimes \chi^{\prime}\right)\left(v_{1}^{\prime} \otimes v_{1}^{\prime \vee}\right)$. On a vu que $f_{\zeta^{\prime}}$ est une fonction lisse à support compact. La proposition 2.4 s'applique à $f_{\zeta}$ et $f_{\zeta^{\prime}}$. Comme $\zeta^{\prime}$ est régulière en tout $\chi^{\prime}$, on obtient

$$
\begin{aligned}
0 & =\int_{G} f_{\zeta}(g) \overline{f_{\zeta^{\prime}}(g)} d g \\
& =\int_{G} \int_{\operatorname{Re}\left(\chi^{\prime}\right)=0} f_{\zeta}(g) \overline{p\left(\sigma^{\prime} \otimes \chi^{\prime}\right)} \overline{j_{1}\left(\sigma^{\prime} \otimes \chi^{\prime}\right)} \overline{\left\langle i_{P^{\prime}}^{G}\left(\sigma^{\prime} \otimes \chi^{\prime}\right)\left(g^{-1}\right) v_{1}^{\prime}, v_{1}^{\prime \vee}\right\rangle} d \chi^{\prime} d g \\
& =\int_{\operatorname{Re}\left(\chi^{\prime}\right)=0} \int_{G} f_{\zeta}(g) \overline{p\left(\sigma^{\prime} \otimes \chi^{\prime}\right)} \overline{j_{1}\left(\sigma^{\prime} \otimes \chi^{\prime}\right)}\left\langle i_{P^{\prime}}^{G}\left(\sigma^{\prime} \otimes \chi^{\prime}\right)(g) v^{\prime}, v^{\prime \vee}\right\rangle d g d \chi^{\prime} \\
& =\int_{\operatorname{Re}\left(\chi^{\prime}\right)=0} \overline{p\left(\sigma^{\prime} \otimes \chi^{\prime}\right)} \overline{j_{1}\left(\sigma^{\prime} \otimes \chi^{\prime}\right)}\left\langle\widehat{f}_{\zeta}\left(\sigma^{\prime} \otimes \chi^{\prime}, P^{\prime}\right) v^{\prime}, v^{\prime \vee}\right\rangle d \chi^{\prime} .
\end{aligned}
$$

Ceci étant vrai pour toute fonction polynomiale $p$ sur $\mathcal{O}^{\prime}$, on en déduit que $\left\langle\widehat{f}_{\zeta}\left(\sigma^{\prime} \otimes \chi^{\prime}, P^{\prime}\right) v^{\prime}, v^{\prime \vee}\right\rangle=0$ pour tout $\chi^{\prime} \in \mathfrak{X}_{\mathrm{im}}^{\mathrm{nr}}\left(M^{\prime}\right)$ et en particulier pour $\chi^{\prime}=1$.

3. Notons $\Theta$ l'ensemble des couples $(P, \mathcal{O})$ formés d'un sous-groupe parabolique semi-standard $P=M U$ et de l'orbite inertielle d'une représentation irréductible cuspidale de $M$. Notons PW $(\Theta)$ l'ensemble des familles $\varphi=\left\{\varphi_{P, \mathcal{O}}\right\}_{(P, \mathcal{O}) \in \Theta}$ dont les composantes sont des applications polynomiales qui vérifient les conditions 1$)$ - 4) du théorème 0.1 .

3.1 Soit $\varphi \in \mathrm{PW}(\Theta)$. Choisissons pour tout couple $(P, \mathcal{O})$ une application polynomiale $\xi_{P, \mathcal{O}}$ vérifiant les conclusions de la proposition 0.2 relatives à $\varphi_{P, \mathcal{O}}$. Notons $\zeta_{P, \mathcal{O}}$ l'application rationnelle donnée par $\zeta_{P, \mathcal{O}}(\sigma)=\left(J_{\bar{P} \mid P}(\sigma)^{-1} \otimes 1\right) \xi_{P, \mathcal{O}}(\sigma)$ pour $\sigma \in \mathcal{O}$.

3.1.1 Proposition. Soient $(P, \mathcal{O}),\left(P^{\prime}, \mathcal{O}^{\prime}\right)$ dans $\Theta$ et $\sigma^{\prime} \in \mathcal{O}^{\prime}$. Alors

$$
\begin{aligned}
& \widehat{f_{\zeta_{P, \mathcal{O}}}}\left(P^{\prime}, \sigma^{\prime}\right) \\
&=\gamma\left(G \mid M^{\prime}\right)^{-1} d\left(\sigma^{\prime}\right)^{-1}\left|\operatorname{Stab}_{\mathfrak{X}_{\mathrm{im}}^{\mathrm{nr}}(M)}\left(\sigma^{\prime}\right)\right| \begin{cases}\varphi_{P^{\prime}, \mathcal{O}^{\prime}}\left(\sigma^{\prime}\right), & \text { si } \mathcal{O}^{\prime} \text { et } \mathcal{O} \text { sont conjugués; } \\
0, & \text { sinon. }\end{cases}
\end{aligned}
$$

Preuve. Le corollaire 2.4.1 prouve que $\widehat{f_{\zeta_{P, \mathcal{O}}}}\left(P^{\prime}, \sigma^{\prime}\right)=0$ si $\mathcal{O}^{\prime}$ et $\mathcal{O}$ ne sont pas conjugués. 
Si $\sigma^{\prime} \in \mathcal{O}$ et $P=P^{\prime}$, l'égalité $\widehat{f_{\zeta_{P, \mathcal{O}}}}\left(P^{\prime}, \sigma^{\prime}\right)=\gamma\left(G \mid M^{\prime}\right)^{-1} d\left(\sigma^{\prime}\right)^{-1} \varphi_{P^{\prime}, \mathcal{O}^{\prime}}\left(\sigma^{\prime}\right)$ résulte immédiatement des propositions 2.3 (ii) et 0.2 .

Si $P \neq P^{\prime}$, celle-ci se déduit de l'identité $\left(J_{P^{\prime} \mid P}\left(\sigma^{\prime}\right) \otimes J_{P \mid P^{\prime}}\left(\sigma^{\prime \vee}\right)^{-1}\right) \varphi_{P, \sigma^{\prime}}=$ $\varphi_{P^{\prime}, \sigma^{\prime}}$.

Si finalement $\sigma^{\prime} \in w \mathcal{O}, w \in W^{G}$, l'identité $(\lambda(w) \otimes \lambda(w)) \widehat{f_{\zeta_{P, \mathcal{O}}}}\left(w^{-1} P^{\prime}, w^{-1} \sigma^{\prime}\right)=$ $\widehat{f_{\zeta_{P, \mathcal{O}}}}\left(P^{\prime}, \sigma^{\prime}\right)$ et ce que l'on vient de prouver dans le cas $\sigma^{\prime} \in \mathcal{O}$ impliquent que $\widehat{f_{\zeta_{P, \mathcal{O}}}}\left(P^{\prime}, \sigma^{\prime}\right)=\varphi_{P^{\prime}, \mathcal{O}^{\prime}}\left(\sigma^{\prime}\right)$.

3.1.2 Corollaire. La fonction $f_{\zeta_{P, \mathcal{O}}}$ ne dépend pas du choix de $\xi_{P, \mathcal{O}}$.

Preuve. Ceci résulte de la proposition 3.1.1, puisque l'ensemble des transformées de Fourier $\left\{f_{\zeta_{P, \mathcal{O}}}\left(P^{\prime}, \sigma^{\prime}\right)\right\}_{\left(P^{\prime}, \sigma^{\prime}\right)}$ détermine la fonction $f_{\zeta_{P, \mathcal{O}}}$ : en effet, il est prouvé dans [BZ] (cf. proposition 2.12) que, pour tout $f \in C_{c}^{\infty}(G), f \neq 0$, il existe une représentation lisse irréductible $\pi$ de $G$ telle que $\pi(f) \neq 0$.

On pourra donc écrire $f_{\varphi_{P, \mathcal{O}}}$ à la place de $f_{\zeta_{P, \mathcal{O}}}$.

3.1.3 Corollaire. L'égalité $f_{\varphi_{P, \mathcal{O}}}=f_{\varphi_{P^{\prime}, \mathcal{O}^{\prime}}}$ vaut si $\mathcal{O}$ et $\mathcal{O}^{\prime}$ sont conjugués.

Preuve. La preuve est analogue à celle du corollaire 3.1.2.

3.2 Lorsque $(P=M U, \mathcal{O}) \in \Theta$, posons $[\mathcal{O}]=\left\{w \sigma \mid w \in W^{G}, \sigma \in \mathcal{O}\right\}, W(M, \mathcal{O})=$ $\{w \in W(M, M) \mid w \mathcal{O}=\mathcal{O}\}, c([\mathcal{O}])=|\mathcal{P}(M)|^{-1}\left|W^{M}\right|\left|W^{G}\right|^{-1}|W(M, \mathcal{O})| \gamma(G \mid M)$ $d(\mathcal{O})\left|\operatorname{Stab}_{\mathfrak{X}_{\mathrm{im}}^{\mathrm{nr}}(M)}(\mathcal{O})\right|^{-1}$ et $f_{\varphi_{[\mathcal{O}]}}=c([\mathcal{O}]) \sum_{\left(P^{\prime}, \mathcal{O}^{\prime}\right) \in \Theta, \mathcal{O}^{\prime} \subseteq[\mathcal{O}]} f_{\varphi_{P^{\prime}, \mathcal{O}^{\prime}}}$.

Il résulte de 3.1.1 que la fonction $f_{\varphi_{[\mathcal{O}]}}$ vérifie les propriétés 3 ) et 4 ) énoncées dans l'introduction. La propriété 5) est une conséquence directe de 2.4.

Après avoir rappelé qu'il résulte de la proposition 2.12 dans [BZ] qu'un élément de $C_{c}^{\infty}(G)$ est déterminé par ses transformées de Fourier (cf. remarque dans la preuve du corollaire 3.1.2), on s'aperçoit que l'on a montré le résultat suivant:

Théorème. Soit $\varphi$ dans $\mathrm{PW}(\Theta)$. La fonction

$$
f_{\varphi}=\sum_{(P, \mathcal{O}) \in \Theta} c([\mathcal{O}]) f_{\varphi_{P, \mathcal{O}}},
$$

est l'unique élément de $C_{c}^{\infty}(G)$ qui vérifie $\widehat{f}_{\varphi}(P, \sigma)=\varphi_{P, \mathcal{O}}(\sigma)$ pour tout $(P, \mathcal{O}) \in$ $\Theta, \sigma \in \mathcal{O}$.

3.2.2 Corollaire. Pour que $\varphi$ soit un élément de $\mathrm{PW}(\Theta)$, il faut et il suffit qu'il existe $f$ dans $C_{c}^{\infty}(G)$, telle que $\varphi_{P, \mathcal{O}}(\sigma)=\widehat{f}(P, \sigma)$ pour tout $(P, \mathcal{O}) \in \Theta, \sigma \in \mathcal{O}$. 


\section{B. Une relation polynomiale}

1.1 Si $(\pi, V)$ est une représentation lisse de $G$ et $P=M U$ un sous-groupe parabolique de $G$, notons $\pi_{P}$ la représentation lisse de $M$ dans le module de Jacquet $V_{P}$ de $V$. Lorsque $U_{1}$ est un sous-groupe ouvert compact de $U$, écrivons $V\left(U_{1}\right)$ pour l'ensemble des éléments $v \in V$ tels que $\int_{U_{1}} \pi(u) v d u=0$. Le noyau $V(U)$ de la projection canonique $j_{P}: V \rightarrow V_{P}$ est la réunion des $V\left(U_{1}\right), U_{1}$ parcourant les sous-groupes ouverts compacts de $U$.

L'ensemble des éléments de $V$ invariants pour l'action par un sous-groupe ouvert $H$ de $G$ sera noté $V^{H}$.

1.2 Soit $\mathcal{O}$ l'orbite inertielle d'une représentation irréductible cuspidale de $M$. Un point $\sigma$ de $\mathcal{O}$ sera dit $W(M, M)$-régulier, si $w \sigma \not \sigma \sigma$ pour tout $w \in W(M, M)$. Rappelons que les fonctions rationnelles $j_{\bar{P} \mid P}, P \in \mathcal{P}(M)$, sont toutes égales à une même fonction, notée $j$, et que tout point $W(M, M)$-régulier de $\mathcal{O}$ est régulier pour $j$.

Un élément $\sigma$ de $\mathcal{O}$ sera dit en position générale s'il vérifie les deux propriétés suivantes:

(i) le caractère central de $\sigma$ est $W(M, M)$-régulier;

(ii) $j(\sigma) \neq 0$.

Fixons $\sigma \in \mathcal{O}$. L'ensemble des $\chi \in \mathfrak{X}^{\mathrm{nr}}(M)$ avec $\sigma \otimes \chi$ en position générale est Zariski dense dans $\mathfrak{X}^{\mathrm{nr}}(M)$. Deux applications rationnelles sur $\mathcal{O}$ sont donc égales dès qu'elles coïncident sur l'ensemble des points en position générale.

1.3 Proposition. Soient $(\sigma, E)$ une représentation irréductible cuspidale de $M$ et $P^{\prime} \in \mathcal{P}(M)$. Supposons $\sigma$ en position générale. Alors l'application

$$
i_{P^{\prime}}^{G} E \longrightarrow \bigoplus_{w \in W(M, M)} w E, \quad v \mapsto \bigoplus_{w \in W(M, M)}\left(\left(J_{P \mid w P^{\prime}}(w \sigma) \lambda(w)\right) v\right)(1)
$$

se factorise $\operatorname{par}\left(i_{P^{\prime}}^{G} E\right)_{P}$. Elle induit un isomorphisme

$$
\left(i_{P^{\prime}}^{G}, \sigma\right)_{P} \longrightarrow \bigoplus_{w \in W(M, M)} w \sigma
$$

Preuve: Le résultat énoncé relatif au module de Jacquet faible dans $[\mathrm{W}]$ au cours de la preuve de V.1.1 se généralise sans problème au cas cuspidal.

2. Soit $B$ l'anneau des fonctions régulières d'une variété algébrique affine complexe. La notion d'une $B$-famille de représentations admissibles a été définie dans [BD]: ce sont les couples $(\pi, V)$ formés d'un $B$-module $V$ et d'un homomorphisme $\pi: G \rightarrow \operatorname{Aut}_{B}(V)$ tels que: pour tout $v \in V$, le stabilisateur de $v$ dans $G$ est un 
sous-groupe ouvert et pour tout sous-groupe ouvert $H$ de $G$, le sous-module des invariants $V^{H}$ est un $B$-module plat de type fini.

Le résultat suivant a été montré par Casselman dans le cas d'une représentation admissible (cf. [Cs] propositions 4.1 .4 et 4.1.6). Sa preuve se généralise au cas d'une $B$-famille de représentations admissibles.

Proposition. Soient $\left(\pi_{B}, V_{B}\right)$ une $B$-famille de représentations admissibles de $G$, $P=M U$ un sous-groupe parabolique semi-standard, et $H$ un sous-groupe ouvert compact de $G$ admettant une décomposition d'Iwahori par rapport à $(P, M)$.

Alors il existe un sous-groupe ouvert compact $U_{1}$ de $U$ tel que $V_{B}^{H} \cap V_{B}(U) \subseteq$ $V_{B}\left(U_{1}\right)$. Les espaces $\left(V_{B}^{H}\right)_{a}:=\pi_{B}\left(1_{H a H}\right) V_{B}$ avec $a \in A_{M}$ positif pour $P$ et vérifiant $a U_{1} a^{-1} \subseteq H \cap U$ sont tous égaux à un même espace, noté $S_{P}^{H}\left(V_{B}\right)$. Le foncteur de Jacquet induit un isomorphisme $S_{P}^{H}\left(V_{B}\right) \rightarrow\left(V_{B}\right)_{P}^{H \cap M}$ de B-modules.

Preuve. Le $B$-module $V_{B}^{H}$ est de type fini grâce à la $B$-admissibilité de $\pi_{B}$. Comme $B$ est noethérien, le module $V_{B}^{H} \cap V_{B}(U)$ est également de type fini. L'existence de $U_{1}$ est alors immédiate.

Pour montrer que le foncteur de Jacquet induit un isomorphisme $\left(V_{B}\right)_{a}^{H} \rightarrow$ $\left(V_{B}\right)_{P}^{H \cap M}$ pour tout $a \in A_{M}$ vérifiant les hypothèses de la proposition, on peut généraliser les arguments de [Cs]: le théorème 3.3 de [Cs] qui est le seul résultat qui utilise l'admissibilité reste valable sans cette hypothèse (cf. [BD] proposition 3.5.2). Quant à l'égalité des espaces $\left(V_{B}\right)_{a}^{H}$, on se ramène comme dans [Cs] proposition 4.1.6 à $\left(V_{B}\right)_{a_{1} a_{2}}^{H} \subseteq\left(V_{B}\right)_{a_{1}}^{H}$. Ces deux espaces sont égaux, puisque $\left(V_{B}\right)_{a_{1}}^{H}$ est isomorphe à $\left(V_{B}\right)_{P}^{H \cap M}$ et que le composé $\left(V_{B}\right)_{a_{1} a_{2}}^{H} \hookrightarrow\left(V_{B}\right)_{a_{1}}^{H} \rightarrow\left(V_{B}\right)_{P}^{H \cap M}$ est également un isomorphisme.

3. Fixons un couple parabolique semi-standard $(P, M)$ et une représentation irréductible cuspidale $(\sigma, E)$ de $M$. Choisissons un sous-groupe ouvert compact $H$ de $G$ admettant une décomposition d'Iwahori par rapport à tout couple parabolique semi-standard. (On peut en trouver aussi petit que l'on veut.)

Notons $B=B_{M}$ l'anneau des fonctions régulières sur la variété algébrique $\mathfrak{X}^{\mathrm{nr}}(M)$. Comme dans [W], on déduit de $(\sigma, E)$ les $B$-familles de représentations admissibles $\left(\sigma_{B}, E_{B}\right)$ et $\left(\pi_{B}, V_{B}\right)=\left(i_{P}^{G} \sigma_{B}, i_{P}^{G} E_{B}\right)$ de $M$ et $G$ respectivement. Rappelons que la $B$-famille algébrique $\left(\sigma_{B}, E_{B}\right)$ est définie par

$E_{B}:=E \otimes_{\mathbb{C}} B \quad$ et $\quad \sigma_{B}(m)(e \otimes b):=\sigma(m) e \otimes b_{m} b, \quad$ pour $m \in M, e \in E$ et $b \in B$,

où, pour $m \in M$, on a noté $b_{m} \in B$ le polynôme défini par

$$
b_{M}(\chi):=\chi(m), \quad \text { pour tout } \chi \in \mathfrak{X}^{\mathrm{nr}}(M) .
$$

La classe d'isomorphie de $\left(\sigma_{B}, E_{B}\right)$ et $\left(\pi_{B}, V_{B}\right)$ ne change donc pas si on remplace $\sigma$ par un élément de sa classe inertielle. On pourra donc écrire $i_{P}^{G} E_{\mathcal{O}, B}$, si on ne veut distinguer aucun élément de $\mathcal{O}$. 
3.1 Pour $\chi \in \mathfrak{X}^{\mathrm{nr}}(M)$, notons $E_{\chi}$ et $V_{\chi}$ respectivement les espaces des représentations $\sigma \otimes \chi$ et $i_{P}^{G}(\sigma \otimes \chi)$. On dispose de morphismes de spécialisation $\operatorname{sp}_{\chi}: E_{B} \rightarrow E_{\chi}$ et $\operatorname{sp}_{\chi}: V_{B} \rightarrow V_{\chi}$ qui commutent avec l'action du groupe. Ainsi, toute application polynomiale sur $\mathfrak{X}^{\mathrm{nr}}(M)$ à valeurs dans $E$ ou $i_{P \cap K}^{K} E$ correspond à un élément de $E_{B}$ ou $V_{B}$, et vice versa. On écrira également $E_{\sigma^{\prime}}, V_{\sigma^{\prime}}$ et $\operatorname{sp}_{\sigma^{\prime}}$ si on ne veut distinguer aucun élément de $\mathcal{O}$.

Le lemme suivant est une conséquence immédiate des définitions:

Lemme. Soit $P^{\prime} \in \mathcal{P}(M)$. L'égalité $\operatorname{sp}_{\chi}\left(S_{P^{\prime}}^{H}\left(V_{B}\right)\right)=S_{P^{\prime}}^{H}\left(V_{\chi}\right)$ vaut pour tout $\chi \in \mathfrak{X}^{\mathrm{nr}}(M)$.

3.2 Pour $b \in B$, notons $b^{\vee}$ l'élément de $B$ qui vérifie $b^{\vee}(\chi)=b\left(\chi^{-1}\right)$. On désignera par $E_{B^{\vee}}^{\vee}$ l'espace $E_{B}^{\vee}$ muni de la structure de $B$-module pour laquelle la multiplication scalaire $B \times E_{B}^{\vee} \rightarrow E_{B}^{\vee}$ est donnée par $\left(b, e_{B}^{\vee}\right) \mapsto b^{\vee} e_{B}^{\vee}$. Le produit de dualité $\langle$,$\rangle sur E \times E^{\vee}$ induit par extension des scalaires une forme $B$-linéaire $M$ équivariante $\langle,\rangle_{B}$ sur $E_{B} \times E_{B}^{\vee}$. On en déduit une forme bilinéaire $G$-équivariante $\langle,\rangle_{B}$ sur $V_{B} \times V_{B^{\vee}}^{\vee}$. Pour $\chi \in \mathfrak{X}^{\mathrm{nr}}(M), \operatorname{sp}_{\chi}\left(\langle,\rangle_{B}\right)$ induit alors par passage au quotient le produit de dualité entre $V_{\chi}$ et $V_{\chi^{-1}}^{\vee^{\chi}}$.

Proposition. Soit $P^{\prime} \in \mathcal{P}(M)$. Les B-modules $S_{P^{\prime}}^{H}\left(V_{B}\right)$ et $S \frac{H}{P^{\prime}}\left(V_{B^{\vee}}^{\vee}\right)$ sont libres de type fini et en dualité par $\langle,\rangle_{B}$.

Preuve. En complétant une base de $E^{H \cap M}$ en une base de $E$, on voit que $E_{B}^{H \cap M}$ est un $B$-module libre de rang égal à la dimension de $E^{H \cap M}$. On sait que $\left(V_{B}\right)_{P^{\prime}}^{H \cap M}$ possède une filtration finie dont les sous-quotients sont des $B$-modules libres isomorphes à $E_{B}^{H \cap M}$. On en déduit que $\left(V_{B}\right)_{P^{\prime}}^{H \cap M}$ est libre de même rang que les espaces $\left(V_{\chi}\right)_{P^{\prime}}^{H \cap M}$ ou $\left(V_{\chi^{-1}}^{\vee}\right)_{P^{\prime}}^{H \cap M}, \chi \in \mathfrak{X}^{\mathrm{nr}}(M)$. Il en est de même pour $\left(V_{B}\right) \frac{H \cap M}{P^{\prime}}$.

Fixons des bases $\left\{v_{i}\right\}_{i \in I}$ et $\left\{v_{i}^{\vee}\right\}_{i \in I}$ de $S_{P^{\prime}}^{H}\left(V_{B}\right)$ et $S \frac{H}{P^{\prime}}\left(V_{B^{\vee}}^{\vee}\right)$. Il suffit de montrer que la matrice $\left(\left\langle v_{i}, v_{j}^{\vee}\right\rangle_{B}\right)_{i, j}$ est inversible, i.e. que son déterminant $d$ appartient à $B^{\times}$. Or, dans le cas contraire $d$ serait contenu dans un idéal maximal $m_{\chi}$ de $B$ correspondant à un point $\chi$ de $\mathfrak{X}^{\mathrm{nr}}(M)$. En spécialisant en $\chi$, il en résulterait que la forme bilinéaire $\operatorname{sp}_{\chi}\left(\langle,\rangle_{B}\right)$ restreinte à $S_{P^{\prime}}^{H}\left(V_{\chi}\right) \times S \frac{H}{P^{\prime}}\left(V_{\chi^{-1}}^{\vee}\right)$ serait dégénérée. Ceci est faux (cf. [Cs] théorème 4.2.4).

3.3 Notons $\mathcal{O}$ l'orbite inertielle de $\sigma$. Rappelons que toute représentation lisse $E^{\prime}$ de $M$ admet une décomposition $E^{\prime}=E^{\prime}(\mathcal{O}) \oplus E^{\prime}($ hors $\mathcal{O})$, telle que tout sousquotient de $E^{\prime}(\mathcal{O})$ soit dans $\mathcal{O}$ et qu'aucun sous-quotient de $E^{\prime}$ (hors $\mathcal{O}$ ) ne le soit.

Pour $P^{\prime} \in \mathcal{P}(M)$, notons $S_{P^{\prime}}^{H}\left(V_{B}\right)(\mathcal{O})$ le sous- $B$-module de $S_{P^{\prime}}^{H}\left(V_{B}\right)$, formé des éléments à image dans $\left(V_{B}\right)_{P^{\prime}}^{H \cap M}(\mathcal{O})$. Définissons de façon analogue $S \frac{H}{P^{\prime}}\left(V_{B^{\vee}}^{\vee}\right)\left(\mathcal{O}^{\vee}\right)$, $S_{P^{\prime}}^{H}\left(V_{\chi}\right)($ hors $\mathcal{O})$ etc. 
3.3.1 Lemme. Les espaces $S_{P^{\prime}}^{H}\left(V_{B}\right)(\mathcal{O})$ et $S_{\frac{H}{P^{\prime}}}\left(V_{B^{\vee}}^{\vee}\right)\left(\right.$ hors $\left.\mathcal{O}^{\vee}\right)$ sont orthogonaux.

Preuve. Soient $v \in S_{P^{\prime}}^{H}\left(V_{B}\right)(\mathcal{O})$ et $v^{\vee} \in S \frac{H}{P^{\prime}}\left(V_{B^{\vee}}^{\vee}\right)\left(\right.$ hors $\left.\mathcal{O}^{\vee}\right)$. Il suffit de montrer que $\operatorname{sp}_{\sigma^{\prime}}\left(\left\langle v, v^{\vee}\right\rangle_{B}\right)=0$, si $\sigma^{\prime} \in \mathcal{O}$ est en position générale. Fixons un tel $\sigma^{\prime}$. On est donc ramené à montrer que $\left\langle v, v^{\vee}\right\rangle=0$ pour $v \in S_{P^{\prime}}^{H}\left(V_{\sigma^{\prime}}\right)(\mathcal{O})$ et $v^{\vee} \in$ $S_{\frac{H}{P^{\prime}}}\left(V_{\sigma^{\prime} \vee}^{\vee}\right)\left(\right.$ hors $\left.\mathcal{O}^{\vee}\right)$. Rappelons (cf. [Cs] paragraphe 4) qu'il existe un produit bilinéaire $M$-équivariant $\langle,\rangle_{P^{\prime}}$ sur $j_{P^{\prime}}\left(V_{\sigma^{\prime}}\right) \times j_{\overline{P^{\prime}}}\left(V_{\sigma^{\prime}}^{\vee}\right)$ tel que

$$
\left\langle v, v^{\vee}\right\rangle=\left\langle j_{P^{\prime}}(v), j_{\overline{P^{\prime}}}\left(v^{\vee}\right)\right\rangle_{P^{\prime}}
$$

Comme, par la proposition $1.3, j_{P^{\prime}}\left(V_{\sigma^{\prime}}\right) \simeq \bigoplus_{w \in W(M, M)} w E_{\sigma^{\prime}}$ et que, par choix de $\sigma^{\prime}, j_{\overline{P^{\prime}}}\left(V_{\sigma^{\prime} \vee}^{\vee}\right) \simeq \bigoplus_{w \in W(M, M)} w E_{\sigma^{\prime} \vee}^{\vee}$, il suffit de considérer le cas $j_{P^{\prime}}(v) \in w E_{\sigma^{\prime}}$ et $j_{\overline{P^{\prime}}}\left(v^{\vee}\right) \in w^{\prime} E_{\sigma^{\vee}}^{\vee}$. Par choix de $v$ et $v^{\vee}$, ou bien un des éléments $j_{P^{\prime}}(v)$ et $j_{\overline{P^{\prime}}}\left(v^{\vee}\right)$ est nul ou bien $w \neq w^{\prime}$. La représentation $\sigma^{\prime}$ étant en position générale, les espaces $w E_{\sigma^{\prime}}$ et $w^{\prime} E_{\sigma^{\prime}}$ n'ont pas d'entrelacement, si $w \neq w^{\prime}$. On en déduit que dans tous les cas $\left\langle j_{P^{\prime}}(v), j_{\overline{P^{\prime}}}\left(v^{\vee}\right)\right\rangle_{P^{\prime}}=0$.

3.3.2 Corollaire. Les B-modules libres $S_{P^{\prime}}^{H}\left(V_{B}\right)(\mathcal{O})$ et $S \frac{H}{P^{\prime}}\left(V_{B^{\vee}}^{\vee}\right)\left(\mathcal{O}^{\vee}\right)$ sont en dualité $\operatorname{par}\langle,\rangle_{B}$.

Preuve. Il suffit de rappeler que $S_{P^{\prime}}^{H}\left(V_{B}\right)=S_{P^{\prime}}^{H}\left(V_{B}\right)(\mathcal{O}) \oplus S_{P^{\prime}}^{H}\left(V_{B}\right)($ hors $\mathcal{O})$.

3.4 On suppose dans cette section que $E^{H \cap M} \neq 0$.

3.4.1 Lemme. Soit $P^{\prime} \in \mathcal{P}(M)$. Tout sous-quotient non nul $V^{\prime}$ de $V_{B}$ en tant que $G$-module vérifie $V_{P^{\prime}}^{\prime}(\mathcal{O}) \neq 0$.

Preuve. D'après $[\mathrm{BD}]$ proposition 2.8 , on a une injection

$$
V^{\prime} \rightarrow \bigoplus_{P^{\prime \prime} \in \mathcal{P}(M)} i_{P^{\prime \prime}}^{G}\left(V_{P^{\prime \prime}}^{\prime}(\mathcal{O})\right)
$$

Par suite, $V_{P^{\prime \prime}}^{\prime}(\mathcal{O}) \neq 0$ pour au moins un $P^{\prime \prime} \in \mathcal{P}(M)$. Ceci équivaut à dire qu'il existe $\sigma^{\prime \prime} \in \mathcal{O}$ avec

$$
0 \neq \operatorname{Hom}_{M}\left(V_{P^{\prime \prime}}^{\prime}, \sigma^{\prime \prime}\right)=\operatorname{Hom}_{G}\left(V^{\prime}, i_{P^{\prime \prime}}^{G} \sigma^{\prime \prime}\right)
$$

Déduisons-en $\sigma^{\prime} \in \mathcal{O}$ avec $\operatorname{Hom}_{M}\left(V_{P^{\prime}}^{\prime}, \sigma^{\prime}\right) \neq 0$ : Par récurrence sur le nombre d'hyperplans radiciels séparant $P^{\prime}$ et $P^{\prime \prime}$, on se ramène à $P^{\prime \prime}$ et $P^{\prime}$ adjacents. Il faut distinguer deux cas:

Si $w P^{\prime \prime} \neq P^{\prime}$ ou $w \sigma^{\prime \prime} \notin \mathcal{O}$ pour tout $w \in W(M, M)$, l'opérateur d'entrelacement $J_{P^{\prime} \mid P^{\prime \prime}}\left(\sigma^{\prime \prime}\right)$ est bien défini et inversible, d'où par composition un élément non nul de $\operatorname{Hom}_{G}\left(V^{\prime}, i_{P^{\prime}}^{G} \sigma^{\prime \prime}\right)=\operatorname{Hom}_{M}\left(V_{P^{\prime}}^{\prime}, \sigma^{\prime \prime}\right)$. 
Dans le cas contraire, choisissons $w \in W(M, M)$ et $\sigma^{\prime} \in \mathcal{O}$ avec $i_{w P^{\prime \prime}}^{G}\left(w \sigma^{\prime \prime}\right)=$ $i_{P^{\prime}}^{G} \sigma^{\prime}$. En composant avec $\lambda(w)$, on trouve donc un élément non nul de $\operatorname{Hom}_{G}\left(V^{\prime}, i_{P}^{G}, \sigma^{\prime}\right)$.

On en déduit que $V_{P^{\prime}}^{\prime}(\mathcal{O}) \neq 0$.

\subsubsection{Proposition.}

Tout sous-G-module $V^{\prime}$ de $V_{B}$ est engendré par $V^{\prime} \cap S_{P^{\prime}}\left(V_{B}^{H}\right)(\mathcal{O})$.

Preuve. Notons $V^{\prime \prime}$ le sous- $G$-module engendré par cet ensemble. On a l'égalité $V_{P^{\prime}}^{\prime \prime}{ }^{H \cap M}(\mathcal{O})=V_{P^{\prime}}^{\prime H \cap M}(\mathcal{O})$, d'où $\left(V^{\prime} / V^{\prime \prime}\right)_{P^{\prime}}^{H \cap M}(\mathcal{O})=0$ par exactitude du foncteur de Jacquet. Comme $H$ a été choisi tel que $E^{H \cap M} \neq 0$, il suit de 3.4 .1 que $V^{\prime}=V^{\prime \prime}$.

4. Rappelons que les ensembles $\Theta$ et $\mathrm{PW}(\Theta)$ ont été définis en A.3. On dira qu'un élément $\varphi$ de $\operatorname{PW}(\Theta)$ a la propriété $(\mathcal{P})$ en $(P, \mathcal{O})$, si $\varphi_{P, \mathcal{O}}$ vérifie les conclusions de la proposition 0.2 . On dira également que $\varphi_{P, \mathcal{O}}$ a la propriété $(\mathcal{P})$.

Fixons pour tout $(P, \mathcal{O}) \in \Theta$ un sous-groupe ouvert compact distingué $H=$ $H(\mathcal{O})$ de $K$ admettant une décomposition d'Iwahori par rapport à tout couple parabolique semi-standard et tel que tout élément de $\mathcal{O}$ admette des invariants par rapport à $H \cap M$. On peut par ailleurs supposer $H\left(\mathcal{O}^{\prime}\right)=H$ si $\mathcal{O}$ et $\mathcal{O}^{\prime}$ sont conjugués, ce que l'on fera désormais.

Pour $\left(P^{\prime}, \mathcal{O}^{\prime}\right) \in \Theta, \mathcal{O}^{\prime}$ conjugué à $\mathcal{O}$, on peut donc parler, grâce à 3.3 .2 , de la projection $\varphi_{P, \mathcal{O}}^{H, P^{\prime}, \mathcal{O}^{\prime}}$ de $i_{P}^{G} E_{\mathcal{O}, B}$ sur $S_{P^{\prime}}^{H}\left(i_{P}^{G} E_{\mathcal{O}, B}\right)\left(\mathcal{O}^{\prime}\right)$ de noyau égal à l'intersection des noyaux des éléments de $S_{\overline{P^{\prime}}}^{H}\left(i_{P}^{G} E_{\mathcal{O}^{\vee}, B^{\vee}}\right)\left(\mathcal{O}^{\vee \vee}\right)$. Si $\left(P^{\prime}, \mathcal{O}^{\prime}\right)=(P, \mathcal{O})$, on écrira plus simplement $\varphi_{P, \mathcal{O}}^{H}$.

4.1 Soit $(P, \mathcal{O}) \in \Theta, H=H(\mathcal{O})$. Rappelons que tout élément $\varphi_{B}$ de $E_{\mathcal{O}, B} \otimes_{B}$ $E_{\mathcal{O}^{\vee}, B^{\vee}}$ ou de $i_{P}^{G} E_{\mathcal{O}, B} \otimes_{B} i_{P}^{G} E_{\mathcal{O}^{\vee}, B^{\vee}}$ correspond par l'application de spécialisation $\mathrm{sp}_{\chi}$ à une application polynomiale sur $\mathfrak{X}^{\mathrm{nr}}(M)$ à valeurs respectivement dans $E_{\mathcal{O}} \otimes E_{\mathcal{O}^{\vee}}$ ou dans $i_{P \cap K}^{K} E_{\mathcal{O}} \otimes i_{P \cap K}^{K} E_{\mathcal{O}^{\vee}}$, et vice versa. On écrira $\varphi_{B}(\sigma)=\operatorname{sp}_{\sigma} \varphi_{B}$. Notons que $\varphi_{P, \mathcal{O}}^{H, P^{\prime}, \mathcal{O}^{\prime}}(\sigma)$ est la projection de $i_{P}^{G} E_{\sigma}$ sur $S_{P^{\prime}}^{H}\left(i_{P}^{G} E_{\sigma}\right)\left(\mathcal{O}^{\prime}\right)$ de noyau égal à l'intersection des noyaux des éléments de $S \frac{H}{P^{\prime}}\left(i_{P}^{G} E_{\sigma^{\vee}}^{\vee}\right)\left(\mathcal{O}^{\prime \vee}\right)$.

4.1.1 Lemme. Soient $P^{\prime \prime} \in \mathcal{P}(M), \sigma \in \mathcal{O}$ et $w \in W^{G}$. Supposons $\sigma$ en position générale. Alors on trouve pour $\left(P^{\prime}, \mathcal{O}^{\prime}\right) \in \Theta, \mathcal{O}^{\prime}$ conjugué à $\mathcal{O}$,

(i) $J_{P^{\prime \prime} \mid P}(\sigma)\left(S_{P^{\prime}}^{H}\left(i_{P}^{G} E_{\sigma}\right)\left(\mathcal{O}^{\prime}\right)\right)=S_{P^{\prime}}^{H}\left(i_{P^{\prime \prime}}^{G} E_{\sigma}\right)\left(\mathcal{O}^{\prime}\right)$;

(ii) $\lambda(w)\left(S_{P^{\prime}}^{H}\left(i_{P}^{G} E_{\sigma}\right)\left(\mathcal{O}^{\prime}\right)\right)=S_{P^{\prime}}^{H}\left(i_{w P}^{G} w E_{\sigma}\right)\left(\mathcal{O}^{\prime}\right)$.

Preuve. Il est clair par définition de $S_{P^{\prime}}^{H}$ que l'image de $S_{P^{\prime}}^{H}\left(i_{P}^{G} E_{\sigma}\right)\left(\mathcal{O}^{\prime}\right)$ par les opérateurs $J_{P^{\prime \prime} \mid P}(\sigma)$ et $\lambda(w)$ est contenu dans $S_{P^{\prime}}^{H}\left(i_{P^{\prime \prime}}^{G} E_{\sigma}\right)$ et $S_{P^{\prime}}^{H}\left(i_{w P}^{G} w E_{\sigma}\right)$ respectivement. Il résulte de la proposition 1.3 que tout sous-quotient de cette image est en fait un élément de $\mathcal{O}^{\prime}$. Ceci prouve l'inclusion. Les opérateurs considérés 
étant bijectifs pour $\sigma$ en position générale, l'inclusion inverse s'en déduit par un argument de symétrie.

4.1.2 Lemme. Avec les notations et hypothèses du lemme 4.1.1, on trouve

(i) $J_{P^{\prime \prime} \mid P}(\sigma) \varphi_{P, \mathcal{O}}^{H, P^{\prime}, \mathcal{O}^{\prime}}(\sigma)=\varphi_{P^{\prime \prime}, \mathcal{O}}^{H, P^{\prime}, \mathcal{O}^{\prime}}(\sigma) J_{P^{\prime \prime} \mid P}(\sigma)$;

(ii) $\lambda(w) \varphi_{P, \mathcal{O}}^{H, P^{\prime}, \mathcal{O}^{\prime}}(\sigma)=\varphi_{w P, w}^{H, P^{\prime}, \mathcal{O}^{\prime}}(w \sigma) \lambda(w)$.

Preuve. Pour $v \in S_{P^{\prime}}^{H}\left(i_{P}^{G} E_{\sigma}\right)\left(\mathcal{O}^{\prime}\right)$, les deux égalités sont immédiates grâce au lemme 4.1.1. Si $v$ est dans le noyau de $\varphi_{P, \mathcal{O}}^{H, P^{\prime}, \mathcal{O}^{\prime}}(\sigma)$, il résulte du lemme 4.1.1 grâce à la propriété d'adjonction de $J_{P^{\prime \prime} \mid P}(\sigma)$ et de $\lambda(w)$ que $J_{P^{\prime \prime} \mid P}(\sigma) v(\operatorname{resp} . \lambda(w) v)$ est un élément du noyau de $\varphi_{P^{\prime \prime}, \mathcal{O}}^{H, P^{\prime}, \mathcal{O}^{\prime}}(\sigma)\left(\operatorname{resp.} \varphi_{w P, w \mathcal{O}}^{H, P^{\prime}, \mathcal{O}^{\prime}}(w \sigma)\right)$.

4.2 Lemme. Pour tout $(P, \mathcal{O}) \in \Theta$, il existe un élément $\varphi$ dans $\mathrm{PW}(\Theta)$ avec $\varphi_{P, \mathcal{O}}=\varphi_{P, \mathcal{O}}^{H(\mathcal{O})}$.

Preuve. Si $\mathcal{O}^{\prime}=w \mathcal{O} w^{-1}$ avec $w \in W$, posons $\varphi_{P^{\prime}, \mathcal{O}^{\prime}}=\varphi_{P^{\prime}, \mathcal{O}^{\prime}}^{H, P, \mathcal{O}}$. Sinon $\varphi_{P^{\prime}, \mathcal{O}^{\prime}}=0$.

Les propriétés 1) et 2) du théorème 0.1 sont vérifiées par définition de $\varphi_{P^{\prime}, \mathcal{O}^{\prime}}^{H, \mathcal{O}^{\prime}}$.

Les propriétés 3) et 4) résultent des égalités (i) et (ii) du lemme 4.1.2.

4.3 Posons $W(M, \mathcal{O})=\{w \in W(M, M) \mid w \mathcal{O}=\mathcal{O}\}$.

Lemme. Supposons: si on a un élément $\varphi$ de $\mathrm{PW}(\Theta)$ et $(P, \mathcal{O})$ vérifiant $\varphi_{P, \mathcal{O}}=$ $\varphi_{P, \mathcal{O}}^{H}$ avec $H=H(\mathcal{O})$, alors cet élément vérifie $(\mathcal{P})$ en $(P, \mathcal{O})$.

Alors la propriété $(\mathcal{P})$ est vérifiée pour tout $\varphi$ de $\mathrm{PW}(\Theta)$ en tout $(P, \mathcal{O})$.

Preuve. Fixons $(P, \mathcal{O})$. Posons $V_{B} \times V_{B^{\vee}}^{\vee}=i_{P}^{G} E_{\mathcal{O}, B} \times i_{P}^{G} E_{\mathcal{O}^{\vee}, B^{\vee}}$. Montrons d'abord que tout élément $\varphi$ de $\mathrm{PW}(\Theta)$ qui vérifie $\varphi_{P, \mathcal{O}} \in S_{P}^{H}\left(V_{B}\right)(\mathcal{O}) \otimes_{B} S_{\bar{P}}^{H}\left(V_{B^{\vee}}^{\vee}\right)\left(\mathcal{O}^{\vee}\right)$ a la propriété $(\mathcal{P})$ en $(P, \mathcal{O})$ :

En effet, de 4.2 et de nos hypothèses, il résulte l'existence d'une application polynomiale $\xi_{P, \mathcal{O}}$ sur $\mathcal{O}$ telle que, pour $\sigma \in \mathcal{O}$,

$$
\varphi_{P, \mathcal{O}}^{H}(\sigma)=\sum_{w \in W(M, \mathcal{O})}\left(J_{P \mid \overline{w P}}(\sigma) \lambda(w) \otimes J_{P \mid w P}\left(\sigma^{\vee}\right) \lambda(w)\right) \xi_{P, \mathcal{O}}\left(w^{-1} \sigma\right) .
$$

On en déduit que

$$
\begin{aligned}
\varphi_{P, \mathcal{O}}(\sigma) & =\varphi_{P, \mathcal{O}}(\sigma) \varphi_{P, \mathcal{O}}^{H}(\sigma) \\
& =\sum_{w \in W(M, \mathcal{O})}\left(J_{P \mid \overline{w P}}(\sigma) \lambda(w) \otimes J_{P \mid w P}\left(\sigma^{\vee}\right) \lambda(w)\right) \varphi_{\bar{P}, \mathcal{O}}\left(w^{-1} \sigma\right) \xi_{P, \mathcal{O}}\left(w^{-1} \sigma\right),
\end{aligned}
$$

le produit dans la première ligne désignant la composition dans l'algèbre $\operatorname{End}\left(i_{P}^{G} E_{\sigma}\right)$. Comme $\sigma \mapsto \varphi \bar{P}, \mathcal{O}\left(w^{-1} \sigma\right)$ est polynomiale, on a une relation du type voulu. 
L'ensemble des éléments $\varphi_{P, \mathcal{O}}$ de $V_{B} \otimes V_{B^{\vee}}^{\vee}$ qui sont la composante en $(P, \mathcal{O})$ d'un élément de $\mathrm{PW}(\Theta)$ est un sous- $G \times G$-module de $V_{B} \otimes V_{B^{\vee}}^{\vee}$. Le sous-ensemble formé des éléments $\varphi_{P, \mathcal{O}}$ qui vérifient par ailleurs la propriété $(\mathcal{P})$ en est un $G \times G$ sous-module. Par ce qui précède, ils ont la même intersection avec $S_{P}^{H}\left(V_{B}\right)(\mathcal{O}) \otimes$ $S \frac{H}{P}\left(V_{B^{\vee}}^{\vee}\right)\left(\mathcal{O}^{\vee}\right)$ qui est non nulle par 4.2. Il résulte alors de 3.4.2 appliqué au sousgroupe parabolique $P \times \bar{P}$ de $G \times G$ et à la représentation de $M \times M$ d'espace $E_{\mathcal{O}, B} \otimes E_{\mathcal{O}^{\vee}, B^{\vee}} \simeq\left(E_{\mathcal{O}} \otimes E_{\mathcal{O}^{\vee}}\right)_{B}$ que ces deux ensembles sont en fait égaux, d'où le lemme.

5. Pour terminer la preuve de la proposition 0.2 , il reste à montrer que $\varphi_{P, \mathcal{O}}^{H(\mathcal{O})}$ a la propriété $(\mathcal{P})$ pour tout $(P, \mathcal{O}) \in \Theta$. Fixons $(P, \mathcal{O})$. Posons $E=E_{\mathcal{O}}$ et $H=H(\mathcal{O})$. On notera parfois, pour $\sigma \in \mathcal{O}$, par $E_{\sigma}$ l'espace $E$ s'il est muni de la représentation $\sigma$.

5.1 Lemme. Soient $\sigma$ dans $\mathcal{O}, P^{\prime} \in \mathcal{P}(M)$ et $v$ un élément non nul de $\left(i_{P^{\prime} \cap K}^{K} E\right)^{H}$ à support dans $\left(P^{\prime} \cap K\right) H$. Soit $a \in A_{M}$ vérifiant les propriétés de la proposition du numéro 2 relatives à $\overline{P^{\prime}}$ et $i_{P^{\prime}}^{G} E_{\sigma}$.

Alors $\left(i_{P^{\prime}}^{G}, \sigma\right)\left(1_{H a H}\right) v$ est un élément non nul de $S_{P^{\prime}}^{H}\left(i_{P^{\prime}}^{G} E_{\sigma}\right)$ à support dans $\left(P^{\prime} \cap K\right) H$ dont la valeur en 1 est proportionelle à $\sigma(a) v(1)$.

Preuve. Notons $v_{\sigma}$ l'élément de $i_{P^{\prime}}^{G} E_{\sigma}$ dont la restriction à $K$ est égal à $v$. Écrivons $P^{\prime}=M U^{\prime}$. Pour $k \in K$, on trouve

$\left(\left(i_{P^{\prime}}^{G} \sigma\right)\left(1_{H a H}\right) v\right)(k)=\frac{\operatorname{mes}(H a H)}{\operatorname{mes}(H)} \int_{H}\left(i_{P^{\prime}}^{G} \sigma(h a) v\right)(k) d h=\frac{\operatorname{mes}(H a H)}{\operatorname{mes}(H)} \int_{H} v_{\sigma}(k h a) d h$.

Pour que $k h a \in \operatorname{supp}\left(v_{\sigma}\right)$, il faut que $k h \in P^{\prime} a H a^{-1}=P^{\prime} a\left(H \cap \overline{U^{\prime}}\right) a^{-1}$. Comme $a$ est positif pour $\overline{P^{\prime}}, a\left(H \cap \overline{U^{\prime}}\right) a^{-1} \subseteq H \cap \overline{U^{\prime}}$, d'où $k \in\left(P^{\prime} \cap K\right) H$. La fonction $\left(i_{P^{\prime}}^{G} \sigma\right)\left(1_{H a H}\right) v$ est donc bien à support dans $\left(P^{\prime} \cap K\right) H$.

Sa valeur en 1 est $\frac{\operatorname{mes}(H a H)}{\operatorname{mes}(H)} \int_{H} v_{\sigma}(h a) d h$. On calcule cette intégrale selon la décomposition $H=\left(H \cap \overline{U^{\prime}}\right)\left(H \cap M^{\prime}\right)\left(H \cap U^{\prime}\right)$. Désignant par $\bar{u}_{P^{\prime}}(h) m_{P^{\prime}}(h) u_{P^{\prime}}(h)$ la décomposition d'un élément $h \in H$ suivant cette décomposition, on trouve $v_{\sigma}(h a)=\delta_{P^{\prime}}^{1 / 2}(a) \sigma(a) v_{\sigma}\left(a^{-1} \bar{u}_{P^{\prime}}(h) a\right)$, puisque $a^{-1} m_{P^{\prime}}(h) u_{P^{\prime}}(h) a \in H$ par choix de $a$. Comme $a^{-1} \bar{u}_{P^{\prime}}(h) a \in \operatorname{supp}\left(v_{\sigma}\right) \subseteq P^{\prime}\left(H \cap \overline{U^{\prime}}\right)$ seulement si $a^{-1} \bar{u}_{P^{\prime}}(h) a \in$ $\left(H \cap \overline{U^{\prime}}\right)$, la deuxième assertion du lemme est immédiate.

5.2 Fixons une base $\left\{e_{i}\right\}_{i \in I}$ de $E^{H \cap M}$. Notons $\left\{e_{i}^{\vee}\right\}_{i \in I}$ la base duale de $\left(E^{\vee}\right)^{H \cap M}$, et $v_{i}\left(\right.$ resp. $\left.v_{i}^{\vee}\right)$ l'élément de $\left(i \frac{K}{P} \cap K=\right)^{H}\left(\operatorname{resp} .\left(i_{P \cap K}^{K} E^{\vee}\right)^{H}\right)$ à support dans $(\bar{P} \cap$ $K) H($ resp. $(P \cap K) H)$ vérifiant $v_{i}(1)=e_{i}\left(\right.$ resp. $\left.v_{i}^{\vee}(1)=e_{i}^{\vee}\right)$.

5.2.1 Lemme. Soit $\sigma$ un point en position générale de $\mathcal{O}$. Alors $v_{i} \in S_{P}^{H}\left(i \frac{G}{P} E_{\sigma}\right)(\mathcal{O})$ 
et de plus, si $w \in W(M, M)$,

$$
\left(\left(J_{P \mid \overline{w P}}(w \sigma) \lambda(w)\right) v_{i}\right)(1)=c_{1} \begin{cases}0, & \text { si } w \neq 1 \\ e_{i}, & \text { si } w=1\end{cases}
$$

où $c_{1}$ est une constante non nulle qui ne dépend pas du choix de $\sigma$.

Preuve. D'après le lemme $5.1, v_{i} \in S_{P}^{H}\left(i \frac{G}{P} E_{\sigma}\right)$. Grâce à la proposition 1.3 , il suffit alors de prouver la dernière assertion. Soit $w \in W(M, M)$. On a

$$
\left(\left(J_{P \mid \overline{w P}}(w \sigma) \lambda(w)\right) v_{i}\right)(1)=\int_{U \cap w U w^{-1}} v_{i, \sigma}\left(w^{-1} u\right) d u,
$$

si cet opérateur d'entrelacement est défini par des intégrales convergentes. Par la décomposition de Bruhat, $w^{-1} u \in \bar{P} H$ seulement si $w=1$. Alors $\int_{U \cap w U w^{-1}} v_{i, \sigma}(u) d u=\operatorname{mes}(H \cap U) e_{i}$. Le cas général s'en déduit par prolongement analytique.

5.3 Lemme. Soit $\sigma \in \mathcal{O}$ en position générale. Les ensembles $\left\{\left(J_{P \mid \overline{w P}}(\sigma) \lambda(w)\right)\right.$ $\left.v_{i}\right\}_{w \in W(M, \mathcal{O}), i \in I}$ et $\left\{\left(J_{P \mid w P}\left(\sigma^{\vee}\right) \lambda(w)\right) v_{i}^{\vee}\right\}_{w \in W(M, \mathcal{O}), i \in I}$ forment des bases de $S_{P}^{H}\left(i_{P}^{G} E_{\sigma}\right)(\mathcal{O})$ et $S_{\bar{P}}^{H}\left(i_{P}^{G} E_{\sigma^{\vee}}^{\vee}\right)\left(\mathcal{O}^{\vee}\right)$ respectivement. On a

$$
\left\langle\left(J_{P \mid \overline{w P}}(\sigma) \lambda(w)\right) v_{i},\left(J_{P \mid w^{\prime} P}\left(\sigma^{\vee}\right) \lambda\left(w^{\prime}\right)\right) v_{j}^{\vee}\right\rangle=c_{2} \delta_{w, w^{\prime}} \delta_{i, j},
$$

où $c_{2}$ est une constante non nulle qui ne dépend pas du choix de $\sigma$.

Preuve. On va utiliser l'isomorphisme de la proposition 1.3. Soit $w^{\prime} \in W(M, \mathcal{O})$. On trouve

$$
\begin{aligned}
\left(J_{P \mid w^{\prime} P}\left(w^{\prime} \sigma\right) \lambda\left(w^{\prime}\right) J_{P \mid \overline{w P}}(\sigma) \lambda\right. & \left.(w) v_{i}\right)(1) \\
& =\left(J_{P \mid w^{\prime} P}\left(w^{\prime} \sigma\right) J_{w^{\prime} P \mid \overline{w^{\prime} w P}}\left(w^{\prime} \sigma\right) \lambda\left(w^{\prime} w\right) v_{i}\right)(1) \\
& =j_{P\left|w^{\prime} P\right| \overline{w^{\prime} w P}}\left(w^{\prime} \sigma\right)\left(J_{P \mid \overline{w^{\prime} w P}}\left(w^{\prime} \sigma\right) \lambda\left(w^{\prime} w\right) v_{i}\right)(1) .
\end{aligned}
$$

Grâce à 5.2.1, ceci n'est non nul que si $w^{\prime}=w^{-1}$, et alors

$\left(J_{P \mid \overline{w^{\prime} w P}}\left(w^{\prime} \sigma\right) \lambda\left(w^{\prime} w\right) v_{i}\right)(1)=c_{1} e_{i} \in w^{-1} E$. Par ailleurs, $j_{P\left|w^{-1} P\right| \bar{P}}\left(w^{-1} \sigma\right)=1$.

On a donc bien une base de $S_{P}^{H}\left(i_{P}^{G} E_{\sigma}\right)(\mathcal{O})$, puisque son image est une base de $\bigoplus_{w \in W(M, \mathcal{O})} w E_{\sigma}$.

Soient $w, w^{\prime} \in W(M, \mathcal{O}), i, j \in I$. Alors

$$
\begin{aligned}
\left\langle\left(J_{P \mid \overline{w P}}(\sigma) \lambda(w)\right)\right. & \left.v_{i},\left(J_{P \mid w^{\prime} P}\left(\sigma^{\vee}\right) \lambda\left(w^{\prime}\right)\right) v_{j}^{\vee}\right\rangle \\
& =\left\langle\lambda\left(w^{\prime-1}\right) J_{w^{\prime} P \mid P}(\sigma) J_{P \mid \overline{w P}}(\sigma) \lambda(w) v_{i}, v_{j}^{\vee}\right\rangle \\
& =\left\langle J_{P \mid w^{\prime-1} P}\left(w^{\prime-1} \sigma\right) J_{w^{\prime-1} P \mid \overline{w^{\prime-1} w P}}\left(w^{\prime-1} \sigma\right) \lambda\left(w^{\prime-1} w\right) v_{i}, v_{j}^{\vee}\right\rangle \\
& =j_{P\left|w^{\prime-1}\right| \overline{w^{\prime-1} w P}}\left(w^{\prime-1} \sigma\right)\left\langle J_{P \mid \overline{w^{\prime-1} w P}}\left(w^{\prime-1} \sigma\right) \lambda\left(w^{\prime-1} w\right) v_{i}, v_{j}^{\vee}\right\rangle .
\end{aligned}
$$


Le support de $v_{j}^{\vee}$ étant $(P \cap K) H$ et l'élément à gauche étant invariant par $H$, ceci n'est non nul que si $\left(J_{P \mid \overline{w^{\prime-1} w P}}\left(w^{\prime-1} \sigma\right) \lambda\left(w^{-1} w\right) v_{i}\right)(1) \neq 0$. Par 5.2.1, ceci n'est possible que si $w=w^{\prime}$. Dans ce cas $j_{P\left|w^{-1} P\right| \bar{P}}\left(w^{-1} \sigma\right)=1$ et l'expression ci-dessus devient par 5.2.1

$\int_{M \cap K} \int_{U \cap K} \int_{H \cap \bar{U}} c_{1}\left\langle\left(w^{-1} \sigma\right)(m) e_{i},\left(w^{-1} \sigma^{\vee}\right)(m) e_{j}^{\vee}\right\rangle d \bar{u} d u d m=\operatorname{mes}(H \cap \bar{U}) c_{1} \delta_{i, j}$, d'où le lemme.

5.4 Posons $\xi_{w}(\sigma)=c_{2}^{-1} \sum_{i \in I} v_{i} \otimes v_{i}^{\vee}$ pour tout $\sigma \in \mathcal{O}$. On vérifie que c'est une application polynomiale sur $\mathcal{O}$. Par ce qui précède, on a

$$
\begin{aligned}
& \sum_{w \in W(M, \mathcal{O})}\left(J_{P \mid \overline{w P}}(\sigma) \lambda(w) \otimes J_{P \mid w P}\left(\sigma^{\vee}\right) \lambda(w)\right) \xi_{w}\left(w^{-1} \sigma\right) \\
= & \sum_{w \in W(M, \mathcal{O})} c_{2}^{-1} \sum_{i \in I}\left(J_{P \mid \overline{w P}}(\sigma) \lambda(w) v_{i} \otimes J_{P \mid w P}\left(\sigma^{\vee}\right) \lambda(w) v_{i}^{\vee}\right) .
\end{aligned}
$$

En se rappelant que l'on a identifié $i_{P}^{G} E_{\sigma} \otimes i_{P}^{G} E_{\sigma^{\vee}}^{\vee}$ à un sous-espace de $\operatorname{End}_{\mathbb{C}}\left(i_{P}^{G} E_{\sigma}\right)$, on déduit de 5.3 que cette expression est égale à $\varphi_{P, \mathcal{O}}^{H}(\sigma)$ pour $\sigma$ en position générale. Par prolongement analytique, ces deux applications sont donc égales.

Lemme. Posons $\xi(\sigma)=|W(M, \mathcal{O})|^{-1} \sum_{w \in W(M, \mathcal{O})} \xi_{w}(\sigma)$. Pour tout $\sigma \in \mathcal{O}$, on a

$$
\varphi_{P, \mathcal{O}}^{H}(\sigma)=\sum_{w \in W(M, \mathcal{O})}\left(J_{P \mid \overline{w P}}(\sigma) \lambda(w) \otimes J_{P \mid w P}\left(\sigma^{\vee}\right) \lambda(w)\right) \xi\left(w^{-1} \sigma\right) .
$$

Preuve. En utilisant les règles de composition pour les opérateurs d'entrelacement, ainsi que les propriétés de $\varphi_{P, \mathcal{O}}^{H}$ relatives aux opérateurs d'entrelacement, on trouve en effet:

$$
\begin{aligned}
& \sum_{w, w^{\prime} \in W(M, \mathcal{O})}\left(J_{P \mid \overline{w P}}(\sigma) \lambda(w) \otimes J_{P \mid w P}\left(\sigma^{\vee}\right) \lambda(w)\right) \xi_{w^{\prime}}\left(w^{-1} \sigma\right) \\
= & \sum_{w, w^{\prime} \in W(M, \mathcal{O})}\left(J_{P \mid \overline{w w^{\prime} P}}(\sigma) \lambda\left(w w^{\prime}\right) \otimes J_{P \mid w w^{\prime} P}\left(\sigma^{\vee}\right) \lambda\left(w w^{\prime}\right)\right) \xi_{w^{\prime}}\left(w^{\prime-1} w^{-1} \sigma\right) \\
= & \sum_{w \in W(M, \mathcal{O})}\left(J_{P \mid w P}(\sigma) \lambda(w) \otimes J_{P \mid w P}\left(\sigma^{\vee}\right) \lambda(w)\right) \sum_{w^{\prime} \in W(M, \mathcal{O})} j_{P|w P| \overline{w w^{\prime} P}}(\sigma)^{-1} \\
= & \sum_{w \in W(M, \mathcal{O})} j_{P \mid w P}(\sigma)^{-1}\left(J_{P \mid w P}(\sigma) \lambda(w) \otimes J_{P \mid w P}\left(\sigma^{\vee}\right) \lambda(w)\right) \varphi_{P, \mathcal{O}}^{H}\left(w^{-1} \sigma\right) \\
= & |W(M, \mathcal{O})| \varphi_{P, \mathcal{O}}^{H}(\sigma) .
\end{aligned}
$$




\section{Références bibliographiques}

[BD] J. N. Bernstein, Le "centre" de Bernstein (rédigé par P.Deligne) dans Représentations des groupes réductifs sur un corps local, par J.N. Bernstein, P. Deligne, D. Kazhdan, M.-F. Vignéras; Travaux en cours, Hermann, Paris 1984.

[BZ] J. Bernstein, A. Zelevinsky, Representations of the group $G L(n, F)$, where $F$ is a local non archimedean field, Uspekhi Mat. Nauk. 313 (1976), 5-70.

[Ca] P. Cartier, Representations of $\wp$-adic groups: a survey, Proc. of Symp. in Pure Mathematics Vol. 33 (1979), part 1, pp. 111-155.

[Cs] W. Casselman, Introduction to the theory of admissible representations of p-adic reductive groups non publié.

[S] A. Silberger, Introduction to Harmonic Analysis on reductive p-adic groups, Mathematical Notes 23, Princeton University Press, Princeton 1979.

[W] J.-L. Waldspurger, La formule de Plancherel pour les groupes p-adiques - d'après HarishChandra à paraître.

\section{Volker Heiermann}

Institut für Mathematik

Humboldt-Universität zu Berlin

Unter den Linden

10099 Berlin

Germany

e-mail: heierman@mathematik.hu-berlin.de

(Received: June 7, 2000) 